\title{
Qualitative and Bifurcation Analysis of an SIR Epidemic Model with Saturated Treatment Function and Nonlinear Pulse Vaccination
}

\author{
Xiangsen Liu ${ }^{1,2}$ and Binxiang Dai ${ }^{1}$ \\ ${ }^{1}$ School of Mathematics and Statistics, Central South University, Changsha, Hunan 410083, China \\ ${ }^{2}$ Department of Mathematics, North University of China, Taiyuan, Shanxi 030051, China
}

Correspondence should be addressed to Binxiang Dai; bxdai@csu.edu.cn

Received 17 April 2016; Revised 26 July 2016; Accepted 16 August 2016

Academic Editor: Seenith Sivasundaram

Copyright (C) 2016 X. Liu and B. Dai. This is an open access article distributed under the Creative Commons Attribution License, which permits unrestricted use, distribution, and reproduction in any medium, provided the original work is properly cited.

An SIR epidemic model with saturated treatment function and nonlinear pulse vaccination is studied. The existence and stability of the disease-free periodic solution are investigated. The sufficient conditions for the persistence of the disease are obtained. The existence of the transcritical and flip bifurcations is considered by means of the bifurcation theory. The stability of epidemic periodic solutions is discussed. Furthermore, some numerical simulations are given to illustrate our results.

\section{Introduction}

The SIR epidemic models have attracted much attention in recent years. In most cases, ordinary differential equations are used to build SIR epidemic models [1-5]. However, impulsive differential equations $[6,7]$ are also suitable for the mathematical simulation of evolutionary processes in which the parameters state variables undergo relatively long periods of smooth variation followed by a short-term rapid change in their values. Many results have been obtained for SIR epidemic models described by impulsive differential equations [8-13].

In the classical epidemic models, it is usually assumed that the removal rate of the infective individuals is proportional to the number of the infective individuals, which implies that the medical resources such as drugs, vaccines, hospital beds, and isolation places are very sufficient for the infectious disease. However, in reality, every community or country has an appropriate or limited capacity for treatment and vaccination.

In order to investigate the effect of the limited capacity for treatment on the spread of infectious disease, Wang and Ruan [14] introduced a constant treatment in an SIR model

$$
h(I)= \begin{cases}r, & I>0, \\ 0, & I=0,\end{cases}
$$

which simulated a limited capacity for treatment. Further, Wang [15] modified the constant treatment to

$$
h(I)= \begin{cases}r I, & 0 \leq I \leq I_{0}, \\ r I_{0}, & I>I_{0},\end{cases}
$$

which meant that the treatment rate was proportional to the number of the infective individuals before the capacity of treatment was reached and then took its maximum value $r I_{0}$. Recently, Zhou and Fan [16] introduced the following continually differentiable treatment function:

$$
h(I)=\frac{\alpha I}{\omega+I},
$$

where $\alpha \geq 0$ represents the maximal medical resources supplied per unit time and $\omega>0$ is half-saturation constant, 
which measures the efficiency of the medical resource supply in the sense that if $\omega$ is smaller, then the efficiency is higher. They investigated the following SIR model:

$$
\begin{aligned}
& S^{\prime}(t)=A-\frac{\beta S I}{1+k I}-d S, \\
& I^{\prime}(t)=\frac{\beta S I}{1+k I}-(d+\gamma+\sigma) I-\frac{\alpha I}{\omega+I}, \\
& R^{\prime}(t)=\gamma I+\frac{\alpha I}{\omega+I}-d R,
\end{aligned}
$$

where $S(t), I(t)$, and $R(t)$ denote the numbers of susceptible, infective, and recovered individuals at time $t$, respectively. $A$ is the recruitment rate of the population, $d$ is the natural death rate of the population, $\gamma$ is the natural recovery rate, and $\sigma$ is the disease-related mortality. The incidence rate $\beta S I /(1+k I)$ is of saturated type and reflects the "psychological" effect or the inhibition effect [17].

In [16], the authors addressed some problems on system (4) such as the existence of endemic equilibria and backward bifurcation, the locally and globally asymptotic stability of the disease-free equilibrium and endemic equilibrium, and the existence of the Hopf bifurcation.

In addition to the treatment, vaccination is often restricted by limited medical resources. The vaccination success rate always has some saturation effect. That is, vaccination rate can be expressed as a saturation function as follows [18]:

$$
p(t)=\frac{q S(t)}{S(t)+\theta} .
$$

Here, $q$ is the maximum pulse immunization rate. $\theta$ is the half-saturation constant, that is, the number of susceptible individuals when the vaccination rate is half the largest vaccination rate. They established the following SIR epidemic model:

$$
\begin{aligned}
S^{\prime}(t) & =a A-\frac{\beta S I}{1+k I}-d S, \\
I^{\prime}(t) & =\frac{\beta S I}{1+k I}-(d+\gamma+\sigma) I, \\
R^{\prime}(t) & =(1-a) A+\gamma I-d R, \\
\Delta S(t) & =-p(t) S(t), \\
\Delta I(t) & =0, \\
\Delta R(t) & =p(t) S(t),
\end{aligned}
$$

$$
t=n T
$$

where $A$ represents the total number of input population. $a(0<a \leq 1)$ is the proportion of input population without immunity.

In [18], the authors addressed some problems on system (6) such as the existence and stability of the disease-free periodic solution of system (6) and the existence of the transcritical bifurcations.

Motivated by $[16,18]$, the following SIR epidemic model with saturated treatment function and nonlinear pulse vaccination is considered:

$$
\begin{aligned}
& S^{\prime}(t)=A-\frac{\beta S I}{1+k I}-d S, \\
& I^{\prime}(t)=\frac{\beta S I}{1+k I}-(d+\gamma+\sigma) I-\frac{\alpha I}{\omega+I}, \\
& R^{\prime}(t)=\gamma I+\frac{\alpha I}{\omega+I}-d R, \\
& t \neq n T \text {, } \\
& \Delta S(t)=-p(t) S(t), \\
& \Delta I(t)=0, \\
& \Delta R(t)=p(t) S(t), \\
& t=n T \text {. }
\end{aligned}
$$

Noticing that variable $R$ just appears in the third and sixth equations of model (7), we only need to consider the following subsystem of model (7):

$$
\begin{aligned}
& S^{\prime}(t)=A-\frac{\beta S I}{1+k I}-d S, \\
& I^{\prime}(t)=\frac{\beta S I}{1+k I}-(d+\gamma+\sigma) I-\frac{\alpha I}{\omega+I}, \\
& t \neq n T \\
& \Delta S(t)=-p(t) S(t), \\
& \Delta I(t)=0, \\
& t=n T .
\end{aligned}
$$

The remaining part of this paper is organized as follows. In the next section, we discuss the existence and stability of the disease-free periodic solution of system (8). In Section 3, the persistence of the disease is considered. In Section 4, the existence of positive periodic solutions is discussed by using the bifurcation theory. We study the stability of the positive periodic solution of system (8) in Section 5. In Section 6, we consider the existence of flip bifurcations by means of the bifurcation theory. In Section 7, some numerical simulations are given to illustrate our results. Finally, some concluding remarks are given.

\section{The Existence and Stability of the Disease-Free Periodic Solution}

In this section, we investigate the existence of the diseasefree periodic solution of system (8). In this case, infectious 
individuals are entirely absent from the population permanently, that is, $I(t)=0, t \geq 0$. System (8) yields

$$
\begin{aligned}
S^{\prime}(t) & =A-d S, \quad t \neq n T, \\
\Delta S(t) & =-p(t) S(t), \quad t=n T .
\end{aligned}
$$

Lemma 1 (see [18]). System (9) has a unique globally asymptotically stable periodic solution $S^{*}(t)$, where

$$
\begin{aligned}
S^{*}(t) & =\frac{A}{d}+\left(\bar{S}-\frac{A}{d}\right) e^{-d(t-n T)}, \quad n T<t \leq(n+1) T, \\
\bar{S} & =\frac{-q_{2}+\sqrt{q_{2}^{2}-4 q_{1} q_{3}}}{2 q_{1}}, \\
q_{1} & =e^{-d T}-(1-q) e^{-2 d T}>0, \\
q_{2} & =\left(e^{-d T}-1\right)\left(\frac{2 A}{d}(1-q) e^{-d T}-\frac{A}{d}-\theta\right), \\
q_{3} & =-(1-q) \frac{A^{2}}{d^{2}}\left(e^{-d T}-1\right)^{2}-\frac{A \theta}{d}\left(1-e^{-d T}\right) \\
& <0 .
\end{aligned}
$$

According to Lemma 1, we obtain the following result.

Theorem 2. System (8) has a disease-free periodic solution $\left(S^{*}(t), 0\right)$.

Next, we will discuss the stability of the periodic solution $\left(S^{*}(t), 0\right)$. The associated eigenvalues of the periodic solution $\left(S^{*}(t), 0\right)$ are

$$
\begin{aligned}
& \lambda_{1}=\left(1-q\left(1-\frac{\theta^{2}}{\left(S^{*}(T)+\theta\right)^{2}}\right)\right) e^{-d T}<1, \\
& \lambda_{2}=\exp \left(\int_{0}^{T} \beta S^{*}(t) \mathrm{d} t-\left(d+\gamma+\sigma+\frac{\alpha}{\omega}\right) T\right) .
\end{aligned}
$$

According to Floquet theory of impulsive differential equation, the periodic solution $\left(S^{*}(t), 0\right)$ is locally asymptotically stable if $\left|\lambda_{1}\right|<1,\left|\lambda_{2}\right|<1$, that is to say,

$$
\begin{aligned}
& \frac{\beta A}{(d+\gamma+\sigma+\alpha / \omega) d} \\
& \quad+\frac{\beta}{(d+\gamma+\sigma+\alpha / \omega) d T}\left(\bar{S}-\frac{A}{d}\right)\left(1-e^{-d T}\right)
\end{aligned}
$$

$$
<1
$$

where $\bar{S}$ is defined in (11). Denote

$$
\begin{aligned}
R_{0}= & \frac{\beta A}{(d+\gamma+\sigma+\alpha / \omega) d} \\
& +\frac{\beta}{(d+\gamma+\sigma+\alpha / \omega) d T}\left(\bar{S}-\frac{A}{d}\right)\left(1-e^{-d T}\right) .
\end{aligned}
$$

Theorem 3. If $R_{0}<1$, then the disease-free periodic solution $\left(S^{*}(t), 0\right)$ of system $(8)$ is locally asymptotically stable.
Remark 4. In [16], the basic reproduction number of system (8) without the pulse vaccination is

$$
R_{0}^{*}=\frac{\beta A}{(d+\gamma+\sigma+\alpha / \omega) d} .
$$

In this paper, the corresponding basic reproduction number of system (8) is

$$
\begin{aligned}
R_{0}= & \frac{\beta A}{(d+\gamma+\sigma+\alpha / \omega) d} \\
& +\frac{\beta}{(d+\gamma+\sigma+\alpha / \omega) d T}\left(\bar{S}-\frac{A}{d}\right)\left(1-e^{-d T}\right) .
\end{aligned}
$$

We claim that $\bar{S}<A / d$. Otherwise, we assume that $\bar{S}>A / d$. By system (9), we find that $S^{*}(T)<\bar{S}$ if $\bar{S}>A / d$. In addition,

$$
S^{*}\left(T^{+}\right)=S^{*}(T)-\frac{q\left(S^{*}(T)\right)^{2}}{S^{*}(T)+\theta}<S^{*}(T)<\bar{S} .
$$

However, $S^{*}(t)$ is a periodic solution with period $T$. It is a contradiction. Thus, $\bar{S}<A / d$. So $R_{0}<R_{0}^{*}$. Hence, the pulse vaccination strategy is beneficial.

Next, we will prove the global attractivity of the diseasefree periodic solution $\left(S^{*}(t), 0\right)$ of system (8).

Define

$$
\begin{aligned}
R_{1}= & \left(d+\gamma+\sigma+\frac{\alpha d}{\omega d+A}\right)^{-1} \\
& \cdot\left(\frac{\beta A}{d}+\frac{\beta}{d T}\left(\bar{S}-\frac{A}{d}\right)\left(1-e^{-d T}\right)\right) .
\end{aligned}
$$

Theorem 5. If $R_{1}<1$, then the disease-free periodic solution $\left(S^{*}(t), 0\right)$ of system $(8)$ is globally attractive.

Proof. If $R_{1}<1$, then $R_{0}<1$. Since $R_{1}<1$, one can choose $\epsilon>0$ small enough such that

$$
\begin{aligned}
\sigma_{1}= & \int_{0}^{T} \beta\left(S^{*}(t)+\epsilon\right) \mathrm{d} t \\
& -\left(d+\gamma+\sigma+\frac{\alpha}{\omega+A / d+\epsilon}\right) T<0 .
\end{aligned}
$$

Let $N(t)=S(t)+I(t)+R(t)$. By system (7), we have

$$
\begin{aligned}
N^{\prime}(t) & =A-d N-\sigma I<A-d N, \quad t \neq n T, \\
\Delta N(t) & =0, \quad t=n T .
\end{aligned}
$$

Consider the following impulsive comparison system:

$$
\begin{aligned}
& x^{\prime}(t)=A-d x, \quad t \neq n T, \\
& \Delta x(t)=0, \quad t=n T .
\end{aligned}
$$

By the comparison theorem of impulsive differential equation, we have $N(t) \leq x(t)$ and $x(t) \rightarrow A / d$ as $t \rightarrow \infty$. Hence, for given $\epsilon$, we have

$$
N(t) \leq x(t)<\frac{A}{d}+\epsilon,
$$


for all $t$ large enough. For simplification, we may assume (23) holds for all $t \geq 0$. It is obvious that, for all $t \geq 0$,

$$
I(t) \leq N(t)<\frac{A}{d}+\epsilon .
$$

From the first and third equations of system (8), we obtain

$$
\begin{gathered}
S^{\prime}(t) \leq A-d S(t), \quad t \neq n T, \\
\Delta S(t)=-p(t) S(t), \quad t=n T .
\end{gathered}
$$

Consider the following impulsive comparison system:

$$
\begin{aligned}
x^{\prime}(t) & =A-d x(t), \quad t \neq n T, \\
\Delta x(t) & =-\frac{q x^{2}(t)}{x(t)+\theta}, \quad t=n T .
\end{aligned}
$$

According to Lemma 1 and the comparison theorem of impulsive differential equation, we get $S(t) \leq x(t)$ and $x(t) \rightarrow$ $S^{*}(t)$ as $t \rightarrow \infty$. Hence, for $\epsilon>0$ and all $t$ large enough

$$
S(t) \leq x(t)<S^{*}(t)+\epsilon .
$$

For simplification, we may suppose (27) holds for all $t \geq 0$.

By the second equation of system (8), we get

$$
I^{\prime}(t)
$$

$$
\begin{aligned}
& \leq\left(\beta\left(S^{*}(t)+\epsilon\right)-\left(d+\gamma+\sigma+\frac{\alpha}{\omega+A / d+\epsilon}\right)\right) \\
& I(t),
\end{aligned}
$$

which leads to

$$
\begin{gathered}
I((n+1) T) \leq I\left(n T^{+}\right) \exp \left(\int_{n T}^{(n+1) T} \beta\left(S^{*}(t)+\epsilon\right) \mathrm{d} t\right. \\
\left.-(d+\gamma+\sigma) T-\frac{\alpha T}{\omega+A / d+\epsilon}\right)=I\left(n T^{+}\right) e^{\sigma_{1}} .
\end{gathered}
$$

Therefore, $I(n T) \leq I\left(0^{+}\right) e^{n \sigma_{1}}$ and $I(n T) \rightarrow 0$ as $n \rightarrow \infty$. From (28), we obtain

$$
\begin{aligned}
& I(t) \leq I\left(n T^{+}\right) \exp \left(\int_{n T}^{t} \beta\left(S^{*}(t)+\epsilon\right) \mathrm{d} t\right. \\
& \left.\quad-(d+\gamma+\sigma) T-\frac{\alpha T}{\omega+A / d+\epsilon}\right) \leq I\left(n T^{+}\right) \\
& \quad \cdot \exp \left(\int_{n T}^{t} \beta\left(S^{*}(t)+\epsilon\right) \mathrm{d} t\right) .
\end{aligned}
$$

Since $S^{*}(t)$ is a periodic solution, there exists a constant $M>$ 0 such that, for all $t \geq 0,\left|S^{*}(t)\right| \leq M$. So $0<I(t) \leq$ $I\left(n T^{+}\right) \exp (\beta(M+\epsilon) T)$. Hence, $I(t) \rightarrow 0$ as $t \rightarrow \infty$. Without loss of generality, we may assume that $0<I(t)<\epsilon$ for $t \geq 0$.

From the first and third equations of system (8), we have

$$
\begin{aligned}
& S^{\prime}(t) \geq A-\left(\frac{\beta \epsilon}{1+k \epsilon}+d\right) S(t), \quad t \neq n T, \\
& \Delta S(t)=-p(t) S(t), \quad t=n T .
\end{aligned}
$$

Consider the following impulsive comparison system:

$$
\begin{aligned}
& x^{\prime}(t)=A-\left(\frac{\beta \epsilon}{1+k \epsilon}+d\right) x(t), \quad t \neq n T, \\
& \Delta x(t)=-\frac{q x^{2}(t)}{x(t)+\theta}, \quad t=n T .
\end{aligned}
$$

By the comparison theorem of impulsive differential equation and Lemma 1, we get

$$
S(t) \geq x(t), \quad x(t) \longrightarrow x^{*}(t)(t \longrightarrow \infty),
$$

where

$$
\begin{aligned}
x^{*}(t) & =\frac{A}{\bar{d}}+\left(\bar{x}-\frac{A}{\bar{d}}\right) e^{-\bar{d}(t-n T)}, \\
\bar{d} & =\frac{\beta \epsilon}{1+k \epsilon}+d, \\
\bar{x} & =\frac{-\bar{q}_{2}+\sqrt{\bar{q}_{2}^{2}-4 \bar{q}_{1} \bar{q}_{3}}}{2 \bar{q}_{1}}, \\
\bar{q}_{1} & =e^{-\bar{d} T}-(1-q) e^{-2 \bar{d} T}>0, \\
\bar{q}_{2} & =\left(e^{-\bar{d} T}-1\right)\left(\frac{2 A}{\bar{d}}(1-q) e^{-\bar{d} T}-\frac{A}{\bar{d}}-\theta\right), \\
\bar{q}_{3} & =-(1-q) \frac{A^{2}}{\bar{d}^{2}}\left(e^{-\bar{d} T}-1\right)^{2}-\frac{A \theta}{\bar{d}}\left(1-e^{-\bar{d} T}\right)
\end{aligned}
$$

$<0$.

By (34), it is easy to see that $x^{*}(t) \rightarrow S^{*}(t)$ as $\epsilon \rightarrow 0$. From (27) and (33), we obtain that, for any $\epsilon_{1}>0$, there exists $T>0$ such that $x^{*}(t)-\epsilon_{1} \leq S(t) \leq S^{*}(t)+\epsilon_{1}$ for $t>T$.

Letting $\epsilon \rightarrow 0$, we have $S^{*}(t)-\epsilon_{1} \leq S(t) \leq S^{*}(t)+\epsilon_{1}$, for $t$ large enough, which implies $S(t) \rightarrow S^{*}(t)$ as $t \rightarrow \infty$. So the disease-free periodic solution $\left(S^{*}(t), 0\right)$ of system $(8)$ is globally attractive. The proof is completed. result.

Synthesizing Theorems 3 and 5, we obtain the following

Theorem 6. The disease-free periodic solution $\left(S^{*}(t), 0\right)$ of system (8) is globally asymptotically stable if $R_{1}<1$.

\section{The Persistence of the Disease}

Theorem 7. If $R_{0}>1$, then there exists a positive constant $m$, such that for any positive solution $I(t)$ of system (8), $\lim \inf _{t \rightarrow \infty} I(t) \geq m$; that is, the disease is uniformly strongly persistent.

Proof. We consider a solution $(S(t), I(t))$ of system (8) and a constant $I^{*} \in(0,1)$. Suppose there exists $t_{0}>0$, such that $I\left(t_{0}\right)<I^{*}$. Let $t_{1}=\sup \left\{s \mid I\left(t_{0}+u\right)<I^{*}, u<s\right\}$. This time interval of length $t_{1}$ can be finite or infinite. Firstly, we prove 
that $t_{1}$ is finite when $I^{*}$ is appropriately chosen. Assume that $t_{1}=\infty$. We see that $I(t)<I^{*}$ holds in the interval $\left[t_{0},+\infty\right)$. Then, from the first and third equations of system (8), we have

$$
\begin{aligned}
S^{\prime}(t) & \geq A-\left(\frac{\beta I^{*}}{1+k I^{*}}+d\right) S(t), \quad t \neq n T, \\
\Delta S(t) & =-p(t) S(t), \quad t=n T .
\end{aligned}
$$

Consider the following impulsive comparison system:

$$
\begin{aligned}
x^{\prime}(t) & =A-\left(\frac{\beta I^{*}}{1+k I^{*}}+d\right) x(t), \quad t \neq n T, \\
\Delta x(t) & =-\frac{q x^{2}(t)}{x(t)+\theta}, \quad t=n T .
\end{aligned}
$$

According to Lemma 1, we obtain that system (37) has a globally asymptotically stable positive periodic solution $x_{1}(t)$, where

$$
\begin{aligned}
x_{1}(t) & =\frac{A}{d_{1}}+\left(S_{2}-\frac{A}{d_{1}}\right) e^{-d_{1}(t-n T)}, \\
& n T<t \leq(n+1) T, \\
d_{1} & =\frac{\beta I^{*}}{1+k I^{*}}+d, \\
S_{2} & =\frac{-p_{2}+\sqrt{p_{2}^{2}-4 p_{1} p_{3}}}{2 p_{1}}, \\
p_{1} & =e^{-d_{1} T}-(1-q) e^{-2 d_{1} T}>0, \\
p_{2} & =\left(e^{-d_{1} T}-1\right)\left(\frac{2 A}{d_{1}}(1-q) e^{-d_{1} T}-\frac{A}{d_{1}}-\theta\right), \\
p_{3} & =-(1-q) \frac{A^{2}}{d_{1}^{2}}\left(e^{-d_{1} T}-1\right)^{2}-\frac{A \theta}{d_{1}}\left(1-e^{-d_{1} T}\right) \\
& <0 .
\end{aligned}
$$

By the comparison principle, there exists $t_{2}>0$ such that, for any $\epsilon>0$ and $t>t_{0}+t_{2}, S(t) \geq x(t) \geq x_{1}(t)-\epsilon$. Meanwhile, $x_{1}(t) \rightarrow S^{*}(t)$ as $I^{*} \rightarrow 0$.

From the second equation of system (8), we obtain

$$
I^{\prime}(t) \geq\left(\frac{\beta\left(x_{1}(t)-\epsilon\right)}{1+k I^{*}}-d-\gamma-\sigma-\frac{\alpha}{\omega}\right) I(t),
$$

for $t>t_{0}+t_{2}$. Let $n_{1} \in Z^{+}, n_{1} T>t_{0}+t_{2}$ and integrating between pulses $[(n-1) T, n T]$ for $n>n_{1}$ yields

$$
\begin{aligned}
& I(n T) \geq I\left((n-1) T^{+}\right) \\
& \cdot \exp \left(\int_{(n-1) T}^{n T}\left(\frac{\beta\left(x_{1}(t)-\epsilon\right)}{1+k I^{*}}-d-\gamma-\sigma-\frac{\alpha}{\omega}\right) \mathrm{d} t\right) .
\end{aligned}
$$

Then we have

$$
\begin{aligned}
& I\left(\left(n_{1}+k\right) T\right) \geq I\left(n_{1} T^{+}\right) \\
& \quad \cdot \exp \left(k \int_{0}^{T}\left(\frac{\beta\left(x_{1}(t)-\epsilon\right)}{1+k I^{*}}-d-\gamma-\sigma-\frac{\alpha}{\omega}\right) \mathrm{d} t\right) .
\end{aligned}
$$

Define $\sigma_{2}=\int_{0}^{T}\left(\beta\left(x_{1}(t)-\epsilon\right) /\left(1+k I^{*}\right)-d-\gamma-\sigma-\alpha / \omega\right) \mathrm{d} t$. Since $x_{1}(t) \rightarrow S^{*}(t)$ as $I^{*} \rightarrow 0$, then $\sigma_{2} \rightarrow \int_{0}^{T}\left(\beta S^{*}(t)-d-\gamma-\right.$ $\sigma-\alpha / \omega) \mathrm{d} t$, as $I^{*} \rightarrow 0$ and $\epsilon \rightarrow 0$. If $I^{*}$ and $\epsilon$ are sufficiently small, then from $R_{0}>1$, we obtain that $\sigma_{2}>0$. Thus, $I\left(\left(n_{1}+\right.\right.$ $k) T) \geq I\left(n_{1} T^{+}\right) e^{k \sigma_{2}} \rightarrow \infty$ as $n \rightarrow \infty$, which contradicts the boundedness of $I(t)$. Therefore, $t_{1}<\infty$ if $I^{*}$ is sufficiently small.

Let us fix these previous $\epsilon$ and $I^{*}$, for which $t_{1}<\infty$. Then we discuss the following two possibilities:

(1) $I(t)>I^{*}$ for all large $t$.

(2) $I(t)$ oscillates about $I^{*}$ for all large $t$.

If possibility (1) holds, then we complete our proof. Next, we will consider possibility (2). From the oscillatory property, we can define an unbounded, monotone increasing sequence $\left\{\tau_{i}\right\}$ such that $\tau_{i}>t_{0}+t_{1}, I\left(\tau_{2 i}\right)<I^{*}$ and $I\left(\tau_{2 i+1}\right) \geq I^{*}$. Choose an arbitrary $\tau_{2 k}$. Let $\tau_{*}=\inf \left\{\tau \mid \tau_{2 k-1}<\tau<\right.$ $\left.\tau_{2 k}, I(\tau) \leq I^{*}\right\}, \bar{\tau}_{*}=\left[\tau_{*} / T\right] T+1-\tau_{*}$, and similarly $\tau^{*}=\sup \left\{\tau \mid \tau_{2 k}<\tau<\tau_{2 k+1}, I(\tau) \leq I^{*}\right\}, \bar{\tau}^{*}=\tau^{*}-\left[\tau^{*} / T\right] T$. Let $l=\min \left\{n \in N \mid \exp \left(-\left(2 T+t_{2}\right)(d+\gamma+\sigma+\alpha / \omega)+n \sigma_{2}\right)>1\right\}$. We see that $\bar{\tau}_{*}<T, \bar{\tau}^{*}<T$, and from the continuity of $I(t)$, we obtain that $I\left(\tau^{*}\right)=I\left(\tau_{*}\right)=I^{*}$. There exists such $l$, because $\sigma_{2}>0$. We claim that $\tau^{*}-\tau_{*}<2 T+t_{2}+l T$. Otherwise, we assume that $\tau^{*}-\tau_{*}>2 T+t_{2}+l T$.

From the second equation of system (8), we have $I^{\prime}(t) \geq$ $-(d+\gamma+\sigma+\alpha / \omega) I(t)$, which gives

$$
\begin{aligned}
& I\left(\tau_{*}+\bar{\tau}_{*}+\bar{\tau}^{*}+t_{2}\right) \\
& \quad \geq I\left(\tau_{*}\right) \exp \left(-\left(\bar{\tau}_{*}+\bar{\tau}^{*}+t_{2}\right)\left(d+\gamma+\sigma+\frac{\alpha}{\omega}\right)\right) \\
& \quad \geq I^{*} \exp \left(-\left(2 T+t_{2}\right)\left(d+\gamma+\sigma+\frac{\alpha}{\omega}\right)\right) .
\end{aligned}
$$

From the assumption $\tau^{*}-\tau_{*}>2 T+t_{2}+l T$, we obtain

$$
\tau_{*}<\tau_{*}+\bar{\tau}_{*}+\bar{\tau}^{*}+t_{2}+l T<\tau_{*}+2 T+t_{2}+l T<\tau^{*} .
$$

Thus, $I\left(\tau_{*}+\bar{\tau}_{*}+\bar{\tau}^{*}+t_{2}+l T\right)<I^{*}$. However, from (40), we get

$$
I\left(\tau_{*}+\bar{\tau}_{*}+\bar{\tau}^{*}+t_{2}+l T\right) \geq I\left(\tau_{*}+\bar{\tau}_{*}+\bar{\tau}^{*}+t_{2}\right) e^{l \sigma_{2}} .
$$

By (42) and (44), we get

$$
\begin{aligned}
& I\left(\tau_{*}+\bar{\tau}_{*}+\bar{\tau}^{*}+t_{2}+l T\right) \\
& \quad \geq I^{*} \exp \left(-\left(2 T+t_{2}\right)\left(d+\gamma+\sigma+\frac{\alpha}{\omega}\right)+l \sigma_{2}\right) \\
& \quad>I^{*}
\end{aligned}
$$

which is a contradiction.

Hence, $\tau^{*}-\tau_{*}<2 T+t_{2}+l T$ and $I(t)>I^{*} \exp (-(2 T+$ $\left.\left.t_{2}+l T\right)(d+\gamma+\sigma+\alpha / \omega)\right)$ for $t \in\left(\tau_{*}, \tau^{*}\right)$. Thus, in case (2), we can set

$$
m=I^{*} \exp \left(-\left(2 T+t_{2}+l T\right)\left(d+\gamma+\sigma+\frac{\alpha}{\omega}\right)\right) .
$$


For any sufficiently large $s$ for which $I(s)<I^{*}$, we have $I(s)>$ $m$, since we can choose $\left\{\tau_{i}\right\}$ such that $s \in\left(\tau_{2 n-1}, \tau_{2 n+1}\right)$ for some $n$.

Finally, if there exists $t_{3}>t_{0}+t_{1}$, such that $I(t)>I^{*}$ for all $t>t_{3}$, then the same $m<I^{*}$ works as well as a lower estimate.

Note that $m$ depends only on the fixed constants $I^{*}$ and $\epsilon$; thus we get strong uniform persistence.

\section{The Existence of Transcritical Bifurcations}

In this section, we will discuss the existence of transcritical bifurcations by means of the bifurcation theory. We let the half-saturation constant $\theta$ be the bifurcation parameter.

4.1. The Poincaré Map. Suppose the disease-free periodic solution $\left(S^{*}(t), 0\right)$ with the initial point $A_{0}(\bar{S}, 0)$ and period $T$ passes through the points $A_{0}$ and $B_{0}\left(S^{*}(T), 0\right)$ at time $T$ and then jumps to the point $A_{1}\left(S^{*}\left(T^{+}\right), 0\right)$ due to vaccination pulse. Thus, $S^{*}\left(T^{+}\right)=\bar{S}$.

Consider another solution $\left(S_{1}(t), I_{1}(t)\right)$ of system (8) with the initial point $A_{k}\left(\bar{S}+S_{k}, I_{k}\right)$. This disturbed trajectory starting from the point $A_{k}$ reaches the point $B_{k}\left(S_{1}(T), I_{1}(T)\right)$ at time $T$ and then jumps to the point $A_{k+1}\left(\bar{S}+S_{k+1}, I_{k+1}\right)$. Thus, $\bar{S}+S_{k+1}=S_{1}\left(T^{+}\right), I_{k+1}=I_{1}\left(T^{+}\right)$.

Denote $S(t)=S_{1}(t)-S^{*}(t), I(t)=I_{1}(t)$ and then $S(0)=$ $S_{k}, I(0)=I_{k}$. Let

$$
\begin{aligned}
F_{1}(S, I) & =A-\frac{\beta S I}{1+k I}-d S, \\
F_{2}(S, I) & =\frac{\beta S I}{1+k I}-(d+\gamma+\sigma) I-\frac{\alpha I}{\omega+I}, \\
F_{3}(S) & =-\frac{q S^{2}}{S+\theta} .
\end{aligned}
$$

Then system (8) may be written as

$$
\begin{aligned}
& S^{\prime}(t)=F_{1}\left(S+S^{*}, I\right)-F_{1}\left(S^{*}, 0\right) \triangleq G_{1}(S, I), \\
& I^{\prime}(t)=F_{2}\left(S+S^{*}, I\right) \triangleq G_{2}(S, I), \quad t \neq n T, \\
& \Delta S(t)=F_{3}\left(S+S^{*}\right)-F_{3}\left(S^{*}\right) \triangleq G_{3}(S), \\
& \Delta I(t)=0
\end{aligned}
$$$$
t=n T \text {. }
$$

By the Taylor expansion, we have

$$
\begin{aligned}
G_{1}(S, I)= & A_{1}(t) S+A_{2}(t) I+A_{3}(t) S I+A_{4}(t) I^{2} \\
& +o\left((|S|+|I|)^{3}\right), \\
G_{2}(S, I)= & B_{1}(t) I+B_{2}(t) S I+B_{3}(t) I^{2} \\
& +o\left((|S|+|I|)^{3}\right), \\
G_{3}(S)= & C_{1}(t) S+C_{2}(t) S^{2}+o\left(|S|^{3}\right),
\end{aligned}
$$

where

$$
\begin{aligned}
& A_{1}(t)=-d, \\
& A_{2}(t)=-\beta S^{*}(t), \\
& A_{3}(t)=-\beta, \\
& A_{4}(t)=\beta k S^{*}(t), \\
& B_{1}(t)=\beta S^{*}(t)-d-\gamma-\sigma-\frac{\alpha}{\omega}, \\
& B_{2}(t)=\beta, \\
& B_{3}(t)=-\beta k S^{*}(t)+\frac{\alpha}{\omega^{2}}, \\
& C_{1}(t)=-q+\frac{q \theta^{2}}{\left(S^{*}(t)+\theta\right)^{2}}, \\
& C_{2}(t)=-\frac{q \theta^{2}}{\left(S^{*}(t)+\theta\right)^{3}} .
\end{aligned}
$$

For $0<t \leq T$, let

$$
\begin{aligned}
S(t)= & a_{1}(t) S_{k}+a_{2}(t) I_{k}+a_{3}(t) S_{k}^{2}+a_{4}(t) S_{k} I_{k} \\
& +a_{5}(t) I_{k}^{2}+o\left(\left(\left|S_{k}\right|+\left|I_{k}\right|\right)^{3}\right), \\
I(t)= & b_{1}(t) S_{k}+b_{2}(t) I_{k}+b_{3}(t) S_{k}^{2}+b_{4}(t) S_{k} I_{k} \\
& +b_{5}(t) I_{k}^{2}+o\left(\left(\left|S_{k}\right|+\left|I_{k}\right|\right)^{3}\right),
\end{aligned}
$$

where

$$
\begin{aligned}
& a_{1}(0)=1, \\
& a_{2}(0)=a_{3}(0)=a_{4}(0)=a_{5}(0)=0, \\
& b_{2}(0)=1, \\
& b_{1}(0)=b_{3}(0)=b_{4}(0)=b_{5}(0)=0 .
\end{aligned}
$$

From systems (48), (49), and (51), we get

$$
\begin{aligned}
& a_{1}(t)=e^{-d t}, \\
& a_{2}(t)=e^{-d t} \int_{0}^{t} e^{d s} A_{2}(s) b_{2}(s) \mathrm{d} s \\
& a_{3}(t)=0, \\
& a_{4}(t)=e^{-d t} \int_{0}^{t} e^{d s}\left(A_{2}(s) b_{4}(s)+A_{3}(s) a_{1}(s)\right. \\
& \left.\cdot b_{2}(s)\right) \mathrm{d} s, \\
& a_{5}(t)=e^{-d t} \int_{0}^{t} e^{d s}\left(A_{2}(s) b_{5}(s)+A_{3}(s) a_{2}(s) b_{2}(s)\right. \\
& \left.+A_{4}(s) b_{2}^{2}(s)\right) \mathrm{d} s \\
& b_{1}(t)=0,
\end{aligned}
$$




$$
\begin{aligned}
& b_{2}(t)=\exp \left(\int_{0}^{t} B_{1}(s) \mathrm{d} s\right) \\
& b_{3}(t)=0 \\
& b_{4}(t)=\exp \left(\int_{0}^{t} B_{1}(s) \mathrm{d} s\right) \int_{0}^{t} \exp \left(-\int_{0}^{s} B_{1}(\tau) \mathrm{d} \tau\right) \\
& \cdot B_{2}(s) a_{1}(s) b_{2}(s) \mathrm{d} s, \\
& b_{5}(t)=\exp \left(\int_{0}^{t} B_{1}(s) \mathrm{d} s\right) \int_{0}^{t} \exp \left(-\int_{0}^{s} B_{1}(\tau) \mathrm{d} \tau\right) \\
& \cdot\left(B_{2}(s) a_{2}(s) b_{2}(s)+B_{3}(s) b_{2}^{2}(s)\right) \mathrm{d} s .
\end{aligned}
$$

It follows from system (51) that

$$
\begin{aligned}
S(T)= & a_{1}(T) S_{k}+a_{2}(T) I_{k}+a_{4}(T) S_{k} I_{k}+a_{5}(T) I_{k}^{2} \\
& +o\left(\left(\left|S_{k}\right|+\left|I_{k}\right|\right)^{3}\right), \\
I(T)= & b_{2}(T) I_{k}+b_{4}(T) S_{k} I_{k}+b_{5}(T) I_{k}^{2} \\
& +o\left(\left(\left|S_{k}\right|+\left|I_{k}\right|\right)^{3}\right), \\
S\left(T^{+}\right)= & S(T)+C_{1}(T) S(T)+C_{2}(T) S^{2}(T) \\
& +o\left(|S(T)|^{3}\right), \\
I\left(T^{+}\right)= & I(T) .
\end{aligned}
$$

Using system (54), we obtain

$$
\begin{aligned}
S\left(T^{+}\right)= & D_{1} S_{k}+D_{2} I_{k}+D_{3} S_{k}^{2}+D_{4} S_{k} I_{k}+D_{5} I_{k}^{2} \\
& +o\left(\left(\left|S_{k}\right|+\left|I_{k}\right|\right)^{3}\right) \\
I\left(T^{+}\right)= & b_{2}(T) I_{k}+b_{4}(T) S_{k} I_{k}+b_{5}(T) I_{k}^{2} \\
& +o\left(\left(\left|S_{k}\right|+\left|I_{k}\right|\right)^{3}\right)
\end{aligned}
$$

where

$$
\begin{aligned}
& D_{1}=\left(1+C_{1}(T)\right) a_{1}(T), \\
& D_{2}=\left(1+C_{1}(T)\right) a_{2}(T), \\
& D_{3}=C_{2}(T) a_{1}^{2}(T), \\
& D_{4}=\left(1+C_{1}(T)\right) a_{4}(T)+2 C_{2}(T) a_{1}(T) a_{2}(T), \\
& D_{5}=\left(1+C_{1}(T)\right) a_{5}(T)+C_{2}(T) a_{2}^{2}(T) .
\end{aligned}
$$

From system (55), the following Poincaré map is obtained:

$$
\begin{aligned}
S_{k+1}= & D_{1} S_{k}+D_{2} I_{k}+D_{3} S_{k}^{2}+D_{4} S_{k} I_{k}+D_{5} I_{k}^{2} \\
& +o\left(\left(\left|S_{k}\right|+\left|I_{k}\right|\right)^{3}\right) \\
I_{k+1}= & b_{2}(T) I_{k}+b_{4}(T) S_{k} I_{k}+b_{5}(T) I_{k}^{2} \\
& +o\left(\left(\left|S_{k}\right|+\left|I_{k}\right|\right)^{3}\right) .
\end{aligned}
$$

4.2. Transcritical Bifurcation. In this subsection, we discuss the existence of a transcritical bifurcation by means of map (57).

The fixed point $(0,0)$ of map (57) corresponds to the disease-free periodic solution $\left(S^{*}(t), 0\right)$ of system $(8)$. The associated eigenvalues of the fixed point $(0,0)$ are given by

$$
\begin{aligned}
\lambda_{1} & =D_{1}=\left(1+C_{1}(T)\right) a_{1}(T)=(1-q \\
& \left.+\frac{q \theta^{2}}{\left(S^{*}(T)+\theta\right)^{2}}\right) e^{-d T}<1, \\
\lambda_{2} & =b_{2}(T)=\exp \left(\frac{\beta}{d}\left(\bar{S}-\frac{A}{d}\right)\left(1-e^{-d T}\right)\right. \\
& \left.+\left(\frac{\beta A}{d}-d-\gamma-\sigma-\frac{\alpha}{\omega}\right) T\right),
\end{aligned}
$$

where $\bar{S}$ is defined in (11).

Denote

$$
\begin{aligned}
\theta_{0} & =\left(\frac{A}{d}-B\right)^{-1}\left(\frac{q_{1} B^{2}}{1-e^{-d T}}-\frac{A^{2}}{d^{2}}(1-q)\left(1-e^{-d T}\right)\right. \\
& \left.+\frac{A B}{d}\left(1-2(1-q) e^{-d T}\right)\right)
\end{aligned}
$$

where

$$
B=\frac{A}{d}+\frac{d}{\beta}\left(1-e^{-d T}\right)^{-1}\left(-\frac{\beta A}{d}+d+\gamma+\sigma+\frac{\alpha}{\omega}\right) T .
$$

If $B>0$ and $\theta_{0}>0$, then $\left.\lambda_{2}\right|_{\theta=\theta_{0}}=1$.

By the above analysis, we find that one of the eigenvalues of the fixed point $(0,0)$ is 1 . An eigenvalue with 1 is associated with a transcritical bifurcation in map (57). Hence, $\left(0,0, \theta_{0}\right)$ is a candidate for a transcritical bifurcation point in map (57).

Define

$$
R_{2}=\left\{\frac{\beta^{2}}{d}\left(e^{-2 d T}-e^{-d T}\right)\left(1-q+\frac{q \theta^{2}}{\left(S^{*}(T)+\theta\right)^{2}}\right) \int_{0}^{T} S^{*}(s) \exp \left(\beta \int_{0}^{s} S^{*}(\tau) \mathrm{d} \tau-\left(\gamma+\sigma+\frac{\alpha}{\omega}\right) s\right) \mathrm{d} s(1\right.
$$




$$
\begin{aligned}
& \left.-\left(1-q+\frac{q \theta^{2}}{\left(S^{*}(T)+\theta\right)^{2}}\right) e^{-d T}\right)^{-1}-\beta^{2} \int_{0}^{T} e^{-d s} \int_{0}^{s} S^{*}(\tau) \exp \left(\int_{0}^{\tau} \beta S^{*}(r) \mathrm{d} r-\left(\gamma+\sigma+\frac{\alpha}{\omega}\right) \tau\right) \mathrm{d} \tau \mathrm{d} s \\
& \left.+\int_{0}^{T}\left(-\beta k S^{*}(s)+\frac{\alpha}{\omega^{2}}\right) \exp \left(\int_{0}^{s} \beta S^{*}(\tau) \mathrm{d} \tau-\left(d+\gamma+\sigma+\frac{\alpha}{\omega}\right) s\right) \mathrm{d} s\right\}\left.\right|_{\theta=\theta_{0}} .
\end{aligned}
$$

Theorem 8. Assume that $\theta_{0}>0, B>0$, where $\theta_{0}, B$ are shown in (59) and (60), respectively.

(1) If $R_{2}<0$, then a supercritical bifurcation occurs at $\theta=\theta_{0}$ in system (8). For some $\varepsilon>0$, system (8) has a positive periodic solution for $\theta \in\left(\theta_{0}, \theta_{0}+\varepsilon\right)$.

(2) If $R_{2}>0$, then a subcritical bifurcation occurs at $\theta=\theta_{0}$ in system (8). For some $\varepsilon>0$, system (8) has a positive periodic solution for $\theta \in\left(\theta_{0}-\varepsilon, \theta_{0}\right)$.

Proof. Let $\theta=\theta_{0}+\theta_{1}$; then map (57) can be rewritten as

$$
\begin{aligned}
& F:\left(\begin{array}{l}
S \\
I
\end{array}\right) \longrightarrow \\
& \left(\begin{array}{cc}
e_{1} & e_{2} \\
0 & 1
\end{array}\right)\left(\begin{array}{l}
S \\
I
\end{array}\right)+\left(\begin{array}{c}
e_{3} \theta_{1} S+e_{4} \theta_{1} I+e_{5} S^{2} \\
f_{1} \theta_{1} I+f_{2} S I+f_{3} I^{2}
\end{array}\right) \\
& +\left(\begin{array}{c}
e_{6} S I+e_{7} I^{2}+o\left(\left(\left|\theta_{1}\right|+|S|+|I|\right)^{3}\right) \\
o\left(\left(\left|\theta_{1}\right|+|S|+|I|\right)^{3}\right)
\end{array}\right),
\end{aligned}
$$

where

$$
\begin{aligned}
& e_{1}=\left.D_{1}\right|_{\theta=\theta_{0}}, \\
& e_{2}=\left.D_{2}\right|_{\theta=\theta_{0}}, \\
& e_{3}=\left.\frac{\partial D_{1}}{\partial \theta}\right|_{\theta=\theta_{0}}, \\
& e_{4}=\left.\frac{\partial D_{2}}{\partial \theta}\right|_{\theta=\theta_{0}}, \\
& e_{5}=\left.D_{3}\right|_{\theta=\theta_{0}}, \\
& e_{6}=\left.D_{4}\right|_{\theta=\theta_{0}}, \\
& e_{7}=\left.D_{5}\right|_{\theta=\theta_{0}}, \\
& f_{1}=\left.\frac{\partial b_{2}(T)}{\partial \theta}\right|_{\theta=\theta_{0}}, \\
& f_{2}=\left.b_{4}(T)\right|_{\theta=\theta_{0}}, \\
& f_{3}=\left.b_{5}(T)\right|_{\theta=\theta_{0}},
\end{aligned}
$$

According to map (62), we may let

$$
J=\left(\begin{array}{cc}
1 & \frac{e_{2}}{1-e_{1}} \\
0 & 1
\end{array}\right)
$$

and use the translation $\left(\begin{array}{l}S \\ I\end{array}\right)=J\left(\begin{array}{l}u \\ v\end{array}\right)$; then map (62) becomes

$$
\begin{aligned}
& \left(\begin{array}{l}
u \\
v
\end{array}\right) \rightarrow \\
& \left(\begin{array}{ll}
e_{1} & 0 \\
0 & 1
\end{array}\right)\left(\begin{array}{l}
u \\
v
\end{array}\right) \\
& +\left(\begin{array}{l}
h_{1}\left(u, v, \theta_{1}\right)+o\left(\left(\left|\theta_{1}\right|+|u|+|v|\right)^{3}\right) \\
h_{2}\left(u, v, \theta_{1}\right)+o\left(\left(\left|\theta_{1}\right|+|u|+|v|\right)^{3}\right)
\end{array}\right)
\end{aligned}
$$

where

$$
\begin{aligned}
h_{1}\left(u, v, \theta_{1}\right) & =E_{1} \theta_{1} u+E_{2} \theta_{1} v+E_{3} u^{2}+E_{4} u v+E_{5} v^{2}, \\
h_{2}\left(u, v, \theta_{1}\right) & =f_{1} \theta_{1} v+f_{2} u v+\left(\frac{e_{2} f_{2}}{1-e_{1}}+f_{3}\right) v^{2}, \\
E_{1} & =e_{3}, \\
E_{2} & =\frac{e_{2}\left(e_{6}-f_{1}\right)}{1-e_{1}}+e_{4}, \\
E_{3} & =e_{5}, \\
E_{4} & =\frac{e_{2}\left(2 e_{5}-f_{2}\right)}{1-e_{1}}+e_{6}, \\
E_{5} & =\frac{e_{2}^{2}\left(e_{5}-f_{2}\right)}{\left(1-e_{1}\right)^{2}}+\frac{e_{2}\left(e_{6}-f_{3}\right)}{1-e_{1}}+e_{7} .
\end{aligned}
$$

Now the center manifold theorem is used to determine the nature of the bifurcations of the fixed point $(0,0)$ at $\theta_{1}=0$. There exists a center manifold for (65) which can be locally represented as follows:

$$
\begin{gathered}
w^{c}(0)=\left\{\left(u, v, \theta_{1}\right) \in R^{3} \mid u=f\left(v, \theta_{1}\right), f(0,0)\right. \\
=0, D f(0,0)=0\} .
\end{gathered}
$$

Letting $u=f\left(v, \theta_{1}\right)=g_{1} v^{2}+g_{2} v \theta_{1}+g_{3} \theta_{1}^{2}+o\left(\left(|v|+\left|\theta_{1}\right|\right)^{3}\right)$, and substituting $u$ into (65) yields $f\left(v+h_{2}\left(u, v, \theta_{1}\right), \theta_{1}\right)=$ $e_{1} u+h_{1}\left(u, v, \theta_{1}\right)$. Equating term of like powers to zero gives $g_{1}=E_{5} /\left(1-e_{1}\right), g_{2}=E_{2} /\left(1-f_{1}\right), g_{3}=0$. Then 
$u=\left(E_{5} /\left(1-e_{1}\right)\right) v^{2}+\left(E_{2} /\left(1-f_{1}\right)\right) v \theta_{1}+o\left(\left(|v|+\left|\theta_{1}\right|\right)^{3}\right)$. Hence, map (65) restricted to the center manifold is given by

$$
\begin{aligned}
& H: v \longrightarrow \\
& \begin{aligned}
v+ & f_{1} \theta_{1} v+\left(\frac{e_{2} f_{2}}{1-e_{1}}+f_{3}\right) v^{2}+o\left(\left(|v|+\left|\theta_{1}\right|\right)^{3}\right) \\
& =v h\left(v, \theta_{1}\right),
\end{aligned}
\end{aligned}
$$

where $h\left(v, \theta_{1}\right)=1+f_{1} \theta_{1}+\left(e_{2} f_{2} /\left(1-e_{1}\right)+f_{3}\right) v+o\left(\left(|v|+\left|\theta_{1}\right|\right)^{2}\right)$. Then we consider the following equation:

$$
h\left(v, \theta_{1}\right)=1 .
$$

We find $\partial h(0,0) / \partial v=e_{2} f_{2} /\left(1-e_{1}\right)+f_{3}$.

Firstly, we consider case (1), that is, $R_{2}<0$. If $R_{2}<0$, then $e_{2} f_{2} /\left(1-e_{1}\right)+f_{3}<0$. Thus, by the implicit function theorem, there exists $\varepsilon>0$ and continuously differentiable function $\bar{\beta}:(-\varepsilon, \varepsilon) \rightarrow R$, such that

$$
h\left(\bar{\beta}\left(\theta_{1}\right), \theta_{1}\right)=1,
$$

where $\left|\theta_{1}\right|<\varepsilon$ and $\bar{\beta}(0)=0$.

Let $v=\bar{\beta}\left(\theta_{1}\right)=\theta_{1} \bar{k}$, where $\bar{k}=\bar{k}\left(\theta_{1}\right)$; then (70) can be written as

$$
f_{1}+\left(\frac{e_{2} f_{2}}{1-e_{1}}+f_{3}\right) \bar{k}+o\left(\left|\theta_{1} \bar{k}\right|+\left|\theta_{1}\right|\right)=0 .
$$

It is easy to see that

$$
f_{1}=\left.\frac{\partial b_{2}(T)}{\partial \theta}\right|_{\theta=\theta_{0}}=\frac{\beta\left(1-e^{-d T}\right)^{2}}{d \sqrt{q_{2}^{2}-4 q_{1} q_{3}}}\left(\frac{A}{d}-\bar{S}\right) .
$$

According to Remark 4, we have $\bar{S}<A / d$. So $f_{1}>0$. Therefore, $f_{1}\left(e_{2} f_{2} /\left(1-e_{1}\right)+f_{3}\right)<0$. Hence, (71) has a positive $\operatorname{root} \bar{k}=\bar{k}\left(\theta_{1}\right)$ if $\theta_{1}$ is small enough. However, $v=\theta_{1} \bar{k}>0$. So, $\theta_{1}>0$. Thus, system (8) undergoes a supercritical bifurcation at $\theta=\theta_{0}$ if $R_{2}<0$.

Similar to the above analysis, we may prove that system (8) undergoes a subcritical bifurcation at $\theta=\theta_{0}$ if $R_{2}>0$. This completes the proof.

\section{The Stability of Epidemic Periodic Solutions}

In the following, we discuss the stability of positive periodic solutions of system (8). According to Theorem 8, system (8) has a positive periodic solution $\left(S_{2}(t), I_{2}(t)\right)$ with the initial point $\bar{A}_{0}\left(S_{2}(0), I_{2}(0)\right)$ and period $T$.

Next, we discuss the local stability of the periodic solution $\left(S_{2}(t), I_{2}(t)\right)$. Suppose that $(S(t), I(t))$ is any solution of system (8). Let

$$
\begin{aligned}
& x(t)=S(t)-S_{2}(t), \\
& y(t)=I(t)-I_{2}(t) .
\end{aligned}
$$

Substituting (73) into (8), we obtain the linearization of system (8) as follows:

$$
\begin{aligned}
x^{\prime}(t) & =A_{51}(t) x(t)+A_{52}(t) y(t), \\
y^{\prime}(t) & =B_{51}(t) x(t)+B_{52}(t) y(t), \\
\Delta \neq n T, & t \neq \\
\Delta x(t) & =A_{53}(t) x(t), \\
\Delta y(t) & =0,
\end{aligned}
$$

$$
t=n T,
$$

where

$$
\begin{aligned}
& A_{51}(t)=-\frac{\beta I_{2}(t)}{1+k I_{2}(t)}-d \\
& A_{52}(t)=-\frac{\beta S_{2}(t)}{\left(1+k I_{2}(t)\right)^{2}}, \\
& A_{53}(t)=-q\left(1-\frac{\theta^{2}}{\left(S_{2}(t)+\theta\right)^{2}}\right) \\
& B_{51}(t)=\frac{\beta I_{2}(t)}{1+k I_{2}(t)}, \\
& B_{52}(t)=\frac{\beta S_{2}(t)}{\left(1+k I_{2}(t)\right)^{2}}-d-\gamma-\sigma-\frac{\alpha \omega}{\left(\omega+I_{2}(t)\right)^{2}} .
\end{aligned}
$$

Let $V(t)=|x(t)|+|y(t)|$. Calculating the upper-right derivative of $V(t)$ along the solution of system (74), we have

$$
\begin{aligned}
& D^{+} V(t)=\operatorname{sgn}(x(t))\left(A_{51}(t) x(t)+A_{52}(t) y(t)\right) \\
& \quad+\operatorname{sgn}(y(t))\left(B_{51}(t) x(t)+B_{52}(t) y(t)\right) \\
& \quad \leq-d|x(t)| \\
& \quad+\left(\frac{2 \beta S_{2}(t)}{\left(1+k I_{2}(t)\right)^{2}}-d-\gamma-\sigma-\frac{\alpha \omega}{\left(\omega+I_{2}(t)\right)^{2}}\right) \\
& \quad \cdot|y(t)| .
\end{aligned}
$$

By (10), for all $t \geq 0$, we have

$$
S^{*}(t) \leq \frac{A}{d}+\left(\bar{S}-\frac{A}{d}\right) e^{-d T} \triangleq M_{1} .
$$

Thus, from (27), we obtain that, for any $\bar{\epsilon}>0$ and all $t$ large enough,

$$
S(t) \leq S^{*}(t)+\bar{\epsilon} \leq M_{1}+\bar{\epsilon} .
$$

According to (24), we obtain that, for all $t$ large enough,

$$
I(t) \leq \frac{A}{d}+\bar{\epsilon} .
$$

Since $\left(S_{2}(t), I_{2}(t)\right)$ is a positive periodic solution of system (8), it is obvious that, by (78) and (79), for all $t \geq 0$, we get 
$S_{2}(t) \leq M_{1}, I_{2}(t) \leq A / d$. In addition, by Theorem 7 , there exists $m>0$ such that, for all $t \geq 0, I_{2}(t) \geq m$.

So

$$
\begin{aligned}
& D^{+} V(t) \leq-d|x(t)| \\
& \quad+\left(\frac{2 \beta M_{1}}{(1+k m)^{2}}-d-\gamma-\sigma-\frac{\alpha \omega}{(\omega+A / d)^{2}}\right)|y(t)| .
\end{aligned}
$$

If

$$
\frac{2 \beta M_{1}}{(1+k m)^{2}}-d-\gamma-\sigma-\frac{\alpha \omega}{(\omega+A / d)^{2}}<0
$$

then we may let

$$
\begin{aligned}
\eta & =\max \left\{-d, \frac{2 \beta M_{1}}{(1+k m)^{2}}-d-\gamma-\sigma-\frac{\alpha \omega}{(\omega+A / d)^{2}}\right\} \\
& <0 .
\end{aligned}
$$

By (77), we have

$$
\begin{aligned}
D^{+} V(t) \leq & \eta V(t), \\
V\left(n T^{+}\right)= & \left|x\left(n T^{+}\right)\right|+\left|y\left(n T^{+}\right)\right| \\
= & \left|\left(1-q\left(1-\frac{\theta^{2}}{\left(S_{2}(n T)+\theta\right)^{2}}\right)\right) x(n T)\right| \\
& +|y(n T)| \leq|x(n T)|+|y(n T)| \\
= & V(n T) .
\end{aligned}
$$

Consider the following impulsive comparison system:

$$
\begin{aligned}
D^{+} V_{1}(t) & =\eta V_{1}(t), \quad t \neq n T, \\
\Delta V_{1}(t) & =0, \quad t=n T .
\end{aligned}
$$

Since $\eta<0$, it is obvious that $0 \leq V(t) \leq V_{1}(t)$ and $V_{1}(t) \rightarrow 0$ as $t \rightarrow \infty$. Therefore $V(t) \rightarrow 0$ as $t \rightarrow \infty$. Correspondingly, $x(t) \rightarrow 0, y(t) \rightarrow 0$ as $t \rightarrow \infty$, where $(x(t), y(t))$ is any solution of system (74). Thus, the positive periodic solution $\left(S_{2}(t), I_{2}(t)\right)$ of system (8) is locally stable if

$$
\frac{2 \beta M_{1}}{(1+k m)^{2}}-d-\gamma-\sigma-\frac{\alpha \omega}{(\omega+A / d)^{2}}<0 .
$$

Synthesizing the above analysis, we obtain the following result.

Theorem 9. If

$$
\frac{2 \beta M_{1}}{(1+k m)^{2}}-d-\gamma-\sigma-\frac{\alpha \omega}{(\omega+A / d)^{2}}<0
$$

where $M_{1}$ and $m$ are shown in (77) and (46), respectively, then the epidemic periodic solution $\left(S_{2}(t), I_{2}(t)\right)$ of system (8) is locally stable.

\section{The Existence of Flip Bifurcations}

In this section, we discuss the existence of flip bifurcations in system (8). According to Section 5, system (8) has a positive periodic solution $\left(S_{2}(t), I_{2}(t)\right)$ with period $T$ and the initial point $\bar{A}_{0}\left(S_{2}(0), I_{2}(0)\right)$.

6.1. The Poincaré Map. To establish a Poincaré map, we choose the Poincaré section $S^{*}=\left\{(S, I) \mid I=I_{2}(0)\right\}$. The nontrivial periodic solution $\left(S_{2}(t), I_{2}(t)\right)$ passes through the points $\bar{A}_{0}$ and $\bar{B}_{0}\left(S_{2}(T), I_{2}(T)\right)$ and then jumps to the point $\bar{A}_{1}\left(S_{2}\left(T^{+}\right), I_{2}\left(T^{+}\right)\right)$due to the pulse vaccination. Thus $S_{2}\left(T^{+}\right)=S_{2}(0), I_{2}\left(T^{+}\right)=I_{2}(0)$.

Consider another solution $\left(S_{3}(t), I_{3}(t)\right)$ with the initial point $\bar{A}_{k}\left(S_{2}(0)+S_{k}, I_{2}(0)\right)$ which is on the Poincaré section $S^{*}$. This disturbed trajectory starting from the point $\bar{A}_{k}$ reaches the point $\bar{B}_{k}\left(S_{3}(T), I_{3}(T)\right)$ at time $T$ and then jumps to the point $\bar{A}_{k+1}\left(S_{2}(0)+S_{k+1}, I_{2}(0)\right)$ which is on the Poincaré section $S^{*}$. Thus $S_{2}(0)+S_{k+1}=S_{3}\left(T^{+}\right), I_{2}(0)=I_{3}\left(T^{+}\right)$.

Denote $S(t)=S_{3}(t)-S_{2}(t), I(t)=I_{3}(t)-I_{2}(t)$ and then $S(0)=S_{k}, I(0)=0$. Let

$$
\begin{aligned}
F_{1}(S, I) & =A-\frac{\beta S I}{1+k I}-d S, \\
F_{2}(S, I) & =\frac{\beta S I}{1+k I}-(d+\gamma+\sigma) I-\frac{\alpha I}{\omega+I}, \\
F_{3}(S) & =-\frac{q S^{2}}{S+\theta} .
\end{aligned}
$$

Then system (8) may be written as

$$
\begin{array}{lr}
S^{\prime}(t)=F_{1}\left(S+S_{2}, I+I_{2}\right)-F_{1}\left(S_{2}, I_{2}\right) \triangleq G_{4}(S, I), & t \neq n T, \\
I^{\prime}(t) & =F_{2}\left(S+S_{2}, I+I_{2}\right)-F_{2}\left(S_{2}, I_{2}\right) \triangleq G_{5}(S, I), \\
\Delta S(t)=F_{3}\left(S+S_{2}\right)-F_{3}\left(S_{2}\right) \triangleq G_{6}(S), & \\
\Delta I(t)=0, & t=n T .
\end{array}
$$

By the Taylor expansion, we have

$$
\begin{aligned}
G_{4}(S, I)= & B_{11}(t) S+B_{12}(t) I+B_{13}(t) S^{2}+B_{14}(t) S I \\
& +B_{15}(t) I^{2}+B_{16}(t) S^{3}+B_{17}(t) S^{2} I \\
& +B_{18}(t) S I^{2}+B_{19}(t) I^{3} \\
& +o\left((|S|+|I|)^{4}\right), \\
G_{5}(S, I)= & D_{11}(t) S+D_{12}(t) I+D_{13}(t) S^{2} \\
& +D_{14}(t) S I+D_{15}(t) I^{2}+D_{16}(t) S^{3} \\
& +D_{17}(t) S^{2} I+D_{18}(t) S I^{2}+D_{19}(t) I^{3} \\
& +o\left((|S|+|I|)^{4}\right), \\
G_{6}(S)= & E_{11}(t) S+E_{12}(t) S^{2}+E_{13}(t) S^{3}+o\left(S^{4}\right),
\end{aligned}
$$


where

$$
\begin{aligned}
& B_{11}(t)=-\frac{\beta I_{2}(t)}{1+k I_{2}(t)}-d, \\
& B_{12}(t)=-\frac{\beta S_{2}(t)}{\left(1+k I_{2}(t)\right)^{2}}, \\
& B_{13}(t)=0 \\
& B_{14}(t)=-\frac{\beta}{\left(1+k I_{2}(t)\right)^{2}}, \\
& B_{15}(t)=\frac{\beta k S_{2}(t)}{\left(1+k I_{2}(t)\right)^{3}}, \\
& B_{16}(t)=B_{17}(t)=0, \\
& B_{18}(t)=\frac{\beta k}{\left(1+k I_{2}(t)\right)^{3}}, \\
& B_{19}(t)=-\frac{\beta k^{2} S_{2}(t)}{\left(1+k I_{2}(t)\right)^{4}}, \\
& D_{11}(t)=\frac{\beta I_{2}(t)}{1+k I_{2}(t)}, \\
& D_{12}(t)=\frac{\beta S_{2}(t)}{\left(1+k I_{2}(t)\right)^{2}}-d-\gamma-\sigma-\frac{\alpha \omega}{\left(\omega+I_{2}(t)\right)^{2}},
\end{aligned}
$$$$
D_{13}(t)=0,
$$$$
D_{14}(t)=\frac{\beta}{\left(1+k I_{2}(t)\right)^{2}},
$$$$
D_{15}(t)=-\frac{\beta k S_{2}(t)}{\left(1+k I_{2}(t)\right)^{3}}+\frac{\alpha \omega}{\left(\omega+I_{2}(t)\right)^{3}},
$$$$
D_{16}(t)=D_{17}(t)=0 \text {, }
$$$$
D_{18}(t)=-\frac{\beta k}{\left(1+k I_{2}(t)\right)^{3}},
$$$$
D_{19}(t)=\frac{\beta k^{2} S_{2}(t)}{\left(1+k I_{2}(t)\right)^{4}}-\frac{\alpha \omega}{\left(\omega+I_{2}(t)\right)^{4}},
$$$$
E_{11}(t)=-q+\frac{q \theta^{2}}{\left(S_{2}(t)+\theta\right)^{2}} \text {, }
$$$$
E_{12}(t)=-\frac{q \theta^{2}}{\left(S_{2}(t)+\theta\right)^{3}},
$$$$
E_{13}(t)=\frac{q \theta^{2}}{\left(S_{2}(t)+\theta\right)^{4}} .
$$$$
\text { For } 0<t \leq T \text {, let }
$$$$
\begin{aligned}
& S(t)=a_{11}(t) S_{k}+a_{12}(t) S_{k}^{2}+a_{13}(t) S_{k}^{3}+o\left(S_{k}^{4}\right), \\
& I(t)=b_{11}(t) S_{k}+b_{12}(t) S_{k}^{2}+b_{13}(t) S_{k}^{3}+o\left(S_{k}^{4}\right),
\end{aligned}
$$

where $a_{11}(0)=a_{12}(0)=a_{13}(0)=0, b_{11}(0)=1, b_{12}(0)=$ $b_{13}(0)=0$, and

$$
\begin{aligned}
& a_{11}(t)=\exp \left(\int_{0}^{t} B_{11}(s) \mathrm{d} s\right) \int_{0}^{t} B_{12}(s) b_{11}(s) \\
& \cdot \exp \left(-\int_{0}^{s} B_{11}(\tau) \mathrm{d} \tau\right) \mathrm{d} s \\
& a_{12}(t)=\exp \left(\int_{0}^{t} B_{11}(s) \mathrm{d} s\right) \int_{0}^{t}\left(B_{12}(s) b_{12}(s)\right. \\
& \left.+B_{14}(s) a_{11}(s) b_{11}(s)+B_{15}(s) b_{11}^{2}(s)\right) \\
& \cdot \exp \left(-\int_{0}^{s} B_{11}(\tau) \mathrm{d} \tau\right) \mathrm{d} s \\
& a_{13}(t)=\exp \left(\int_{0}^{t} B_{11}(s) \mathrm{d} s\right) \int_{0}^{t}\left(B_{12}(s) b_{13}(s)\right. \\
& +B_{14}(s)\left(a_{11}(s) b_{12}(s)+a_{12}(s) b_{11}(s)\right) \\
& +2 B_{15}(s) b_{11}(s) b_{12}(s)+B_{18}(s) a_{11}(s) b_{11}^{2}(s) \\
& \left.+B_{19}(s) b_{11}^{3}(s)\right) \exp \left(-\int_{0}^{s} B_{11}(\tau) \mathrm{d} \tau\right) \mathrm{d} s, \\
& b_{11}(t)=\exp \left(\int_{0}^{t} D_{12}(s) \mathrm{d} s\right) \int_{0}^{t} D_{11}(s) a_{11}(s) \\
& \cdot \exp \left(-\int_{0}^{s} D_{12}(\tau) \mathrm{d} \tau\right) \mathrm{d} s, \\
& b_{12}(t)=\exp \left(\int_{0}^{t} D_{12}(s) \mathrm{d} s\right) \int_{0}^{t}\left(D_{11}(s) a_{12}(s)\right. \\
& \left.+D_{14}(s) a_{11}(s) b_{11}(s)+D_{15}(s) b_{11}^{2}(s)\right) \\
& \cdot \exp \left(-\int_{0}^{s} D_{12}(\tau) \mathrm{d} \tau\right) \mathrm{d} s, \\
& b_{13}(t)=\exp \left(\int_{0}^{t} D_{12}(s) \mathrm{d} s\right) \int_{0}^{t}\left(D_{11}(s) a_{13}(s)\right. \\
& +D_{14}(s)\left(a_{11}(s) b_{12}(s)+a_{12}(s) b_{11}(s)\right) \\
& +2 D_{15}(s) b_{11}(s) b_{12}(s)+D_{18}(s) a_{11}(s) b_{11}^{2}(s) \\
& \left.+D_{19}(s) b_{11}^{3}(s)\right) \exp \left(-\int_{0}^{s} D_{12}(\tau) \mathrm{d} \tau\right) \mathrm{d} s .
\end{aligned}
$$

It follows from system (89) and (91) that

$$
\begin{aligned}
S(T) & =a_{11}(T) S_{k}+a_{12}(T) S_{k}^{2}+a_{13}(T) S_{k}^{3}+o\left(S_{k}^{4}\right), \\
I(T) & =b_{11}(T) S_{k}+b_{12}(T) S_{k}^{2}+b_{13}(T) S_{k}^{3}+o\left(S_{k}^{4}\right), \\
S\left(T^{+}\right) & =S(T)+G_{6}(T) \\
& =d_{1} S_{k}+d_{2} S_{k}^{2}+d_{3} S_{k}^{3}+o\left(S_{k}^{4}\right),
\end{aligned}
$$


where

$$
\begin{aligned}
d_{1}= & \left(1+E_{11}(T)\right) a_{11}(T), \\
d_{2}= & \left(1+E_{11}(T)\right) a_{12}(T)+E_{12}(T) a_{11}^{2}(T), \\
d_{3}= & \left(1+E_{11}(T)\right) a_{13}(T)+2 E_{12}(T) a_{11}(T) a_{12}(T) \\
& +E_{13}(T) a_{11}^{3}(T) .
\end{aligned}
$$

By means of (94), the following Poincaré map is obtained:

$$
S_{k+1}=d_{1} S_{k}+d_{2} S_{k}^{2}+d_{3} S_{k}^{3}+o\left(S_{k}^{4}\right)
$$

6.2. Flip Bifurcation. In this subsection, we discuss the existence of a flip bifurcation by means of the Poincaré map (96) and the following lemma.

Lemma 10 (see [19]). Let $f_{\mu}: R \rightarrow R$ be a one-parameter family of map such that $f_{\mu_{0}}$ has a fixed point $x_{0}$ with eigenvalue -1 . Assume the following conditions:

(F1) $\left((\partial f / \partial \mu)\left(\partial^{2} f / \partial x^{2}\right)+2\left(\partial^{2} f / \partial x \partial \mu\right)\right) \neq 0$ at $\left(x_{0}, \mu_{0}\right)$.

$(\mathrm{F} 2) \bar{a}=(1 / 2)\left(\partial^{2} f / \partial x^{2}\right)^{2}+(1 / 3)\left(\partial^{3} f / \partial x^{3}\right) \neq 0$ at $\left(x_{0}, \mu_{0}\right)$.

Then there is a smooth curve of fixed points of $f_{\mu}$ passing through $\left(x_{0}, \mu_{0}\right)$, the stability of which changes at $\left(x_{0}, \mu_{0}\right)$. There is also a smooth curve $\gamma$ passing through $\left(x_{0}, \mu_{0}\right)$ so that $\gamma \backslash\left(x_{0}, \mu_{0}\right)$ is a union of hyperbolic period-two orbits.

In (F2), the sign of $\bar{a}$ determines the stability and direction of bifurcation of the orbits of period two. If $\bar{a}$ is positive, the orbits are stable; if $\bar{a}$ is negative, they are unstable.

The fixed point $S_{k}=0$ of map (96) corresponds to the periodic solution $\left(S_{2}(t), I_{2}(t)\right)$ of system (8). The eigenvalue of map (96) is given by

$$
\begin{aligned}
\lambda & =d_{1}=\left(1+E_{11}(T)\right) a_{11}(T) \\
& =\left(1-q+\frac{q \theta^{2}}{\left(S_{2}(t)+\theta\right)^{2}}\right) a_{11}(T) .
\end{aligned}
$$

Setting $\lambda=-1$, then

$$
\left(1-q+\frac{q \theta^{2}}{\left(S_{2}(T)+\theta\right)^{2}}\right) a_{11}(T)=-1
$$

Define $g(\theta)=1-q+q \theta^{2} /\left(S_{2}(T)+\theta\right)^{2}$. Thus $g(0)=1-$ $q$, $\lim _{\theta \rightarrow \infty} g(\theta)=1$. In addition, it follows from (98) that $a_{11}(T)<0$. Therefore, there exists a positive $\operatorname{root} \theta_{1}$ of (98) if $-(1-q)^{-1}<a_{11}(T)<-1$. An eigenvalue with -1 is associated with a flip bifurcation in map (96). Hence, $\left(0, \theta_{1}\right)$ is a candidate for a flip bifurcation point in map (96).
Theorem 11. Assume that the conditions

$$
\begin{aligned}
& -(1-q)^{-1}<a_{11}(T)<-1, \\
& \left.\frac{\partial a_{11}(T)}{\partial \theta}\right|_{\theta=\theta_{1}} \neq 2 q \theta_{1}\left(\left.S_{2}(T)\right|_{\theta=\theta_{1}}\right. \\
& \left.-\left.\theta_{1} \frac{\partial S_{2}(T)}{\partial \theta}\right|_{\theta=\theta_{1}}\right)\left(\left.S_{2}(T)\right|_{\theta=\theta_{1}}+\theta_{1}\right)^{-3}(1-q \\
& \left.+\frac{q \theta_{1}^{2}}{\left(\left.S_{2}(T)\right|_{\theta=\theta_{1}}+\theta_{1}\right)^{2}}\right)^{-2} \\
& \left.\left(1-q+\frac{q \theta_{1}^{2}}{\left(\left.S_{2}(T)\right|_{\theta=\theta_{1}}+\theta_{1}\right)^{2}}\right) a_{12}^{2}(T)\right|_{\theta=\theta_{1}} \\
& +\left.a_{13}(T)\right|_{\theta=\theta_{1}} \neq q(1-q) \theta_{1}^{2}\left(\left.(1-q) S_{2}(T)\right|_{\theta=\theta_{1}}\right. \\
& \left.+\theta_{1}+\frac{q \theta_{1}^{2}}{\left.S_{2}(T)\right|_{\theta=\theta_{1}}+\theta_{1}}\right)^{-4}
\end{aligned}
$$

hold. Then a flip bifurcation occurs at $\theta=\theta_{1}$ in system (8).

Proof. Let $\theta=\theta_{1}+\theta_{2}$; then map (96) can be rewritten as

$$
\begin{aligned}
f: u \longrightarrow & -u+k_{1} \theta_{2} u+k_{2} \theta_{2}^{2} u+k_{3} u^{2}+k_{4} \theta_{2} u^{2} \\
& +k_{5} u^{3}+o\left(u^{4}\right)
\end{aligned}
$$

where

$$
\begin{aligned}
& k_{1}=\left.\frac{\partial d_{1}}{\partial \theta}\right|_{\theta=\theta_{1}}, \\
& k_{2}=\left.\frac{1}{2} \frac{\partial^{2} d_{1}}{\partial \theta^{2}}\right|_{\theta=\theta_{1}}, \\
& k_{3}=\left.d_{2}\right|_{\theta=\theta_{1}}, \\
& k_{4}=\left.\frac{\partial d_{2}}{\partial \theta}\right|_{\theta=\theta_{1}}, \\
& k_{5}=\left.d_{3}\right|_{\theta=\theta_{1}} .
\end{aligned}
$$

By means of (102), we get $\left(\partial f / \partial \theta_{2}\right)(0,0)\left(\partial^{2} f / \partial u^{2}\right)(0,0)+$ $2\left(\partial^{2} f / \partial u \partial \theta_{2}\right)(0,0)=2 k_{1}$. It is easy to see that

$$
\begin{aligned}
k_{1} & =\left.\frac{\partial d_{1}}{\partial \theta}\right|_{\theta=\theta_{1}} \\
& =\left\{\left(\frac{2 q \theta\left(S_{2}(T)-\theta\left(\partial S_{2}(T) / \partial \theta\right)\right)}{\left(S_{2}(T)+\theta\right)^{3}}\right) a_{11}(T)\right. \\
& \left.+\left(1-q+\frac{q \theta^{2}}{\left(S_{2}(T)+\theta\right)^{2}}\right) \frac{\partial a_{11}(T)}{\partial \theta}\right\}\left.\right|_{\theta=\theta_{1}} .
\end{aligned}
$$




$$
\begin{aligned}
& \text { Since }\left.\left(1-q+q \theta^{2} /\left(S_{2}(T)+\theta\right)^{2}\right) a_{11}(T)\right|_{\theta=\theta_{1}}=-1 \text {, } \\
& \qquad\left.a_{11}(T)\right|_{\theta=\theta_{1}}=-\left(1-q+\frac{q \theta_{1}^{2}}{\left(\left.S_{2}(T)\right|_{\theta=\theta_{1}}+\theta_{1}\right)^{2}}\right)^{-1} .
\end{aligned}
$$

Then

$$
\begin{gathered}
\left.k_{1}=-\frac{2 q \theta_{1}\left(\left.S_{2}(T)\right|_{\theta=\theta_{1}}-\left.\theta_{1}\left(\partial S_{2}(T) / \partial \theta\right)\right|_{\theta=\theta_{1}}\right)}{\left(\left.S_{2}(T)\right|_{\theta=\theta_{1}}+\theta_{1}\right)^{3}}\right)^{-1} 1 \\
\left.-q+\frac{q \theta_{1}^{2}}{\left(\left.S_{2}(T)\right|_{\theta=\theta_{1}}+\theta_{1}\right)^{2}}\right)^{(1-q} \\
\left.+\frac{q \theta_{1}^{2}}{\left(\left.S_{2}(T)\right|_{\theta=\theta_{1}}+\theta_{1}\right)^{2}}\right)\left.\frac{\partial a_{11}(T)}{\partial \theta}\right|_{\theta=\theta_{1}} .
\end{gathered}
$$

It is obvious that $k_{1} \neq 0$ if condition (100) holds.

From map (102), we have $\bar{a}=(1 / 2)\left(\left(\partial^{2} f / \partial u^{2}\right)(0,0)\right)^{2}+$ $(1 / 3)\left(\partial^{3} f / \partial u^{3}\right)(0,0)=2\left(k_{3}^{2}+k_{5}\right)$. By calculation, we obtain

$$
\begin{aligned}
& k_{3}^{2}+k_{5}=\left(1+E_{11}(T)\right) \\
& \cdot\left(\left.\left(1+E_{11}(T)\right) a_{12}^{2}(T)\right|_{\theta=\theta_{1}}+\left.a_{13}(T)\right|_{\theta=\theta_{1}}\right) \\
& \quad-\left.\frac{q \theta^{2}(1-q)}{\left(1+E_{11}(T)\right)^{4}\left(S_{2}(T)+\theta\right)^{4}}\right|_{\theta=\theta_{1}} .
\end{aligned}
$$

So $\bar{a}=2\left(k_{3}^{2}+k_{5}\right) \neq 0$ if condition (101) holds.

Then conditions (F1) and (F2) of Lemma 10 hold. So a flip bifurcation occurs in view of Lemma 10. A $2 T$-periodic solution bifurcates from the $T$-periodic solution at $\theta=$ $\theta_{1}$.

\section{Numerical Simulation}

In this section, we will give bifurcation diagrams and phase portraits of system (8) to illustrate the above theoretical analyses and find new interesting complex dynamical behaviors by using numerical simulations. The bifurcation parameters are considered in the following two cases.

(1) Consider the following set of parameters:

$$
\begin{aligned}
& A=76.5, \\
& \beta=0.5, \\
& k=0.01, \\
& d=0.5, \\
& T=1, \\
& \gamma=7.3, \\
& \sigma=0.2, \\
& \alpha=50,
\end{aligned}
$$

$$
\begin{aligned}
\omega & =\frac{1}{0.3} \\
q & =0.8 .
\end{aligned}
$$

The solution of system (8) from the initial point $(15.5,5.2)$ with $\theta=3$ tends to the stable disease-free periodic solution when $t$ increases (see Figure 1).

By (59), we obtain

$$
\begin{aligned}
\theta_{0} & =\left(\frac{76.5}{0.5}-17.04\right)^{-1}\left(\frac{0.5329 \times(17.04)^{2}}{1-0.6065}\right. \\
& -\frac{(76.5)^{2}}{0.25}(1-0.8)(1-0.6065) \\
& \left.+\frac{76.5 \times 17.04}{0.5}(1-2(1-0.8) \times 0.6065)\right) \\
& \approx 3.866 .
\end{aligned}
$$

The bifurcation diagram of system (8) with respect to $\theta$ is presented in Figure 2. It is seen from the bifurcation diagram that the disease-free periodic solution is stable for $\theta \in(0,3.866)$ and unstable for $\theta \in(3.866,+\infty)$. A positive $T$-periodic solution bifurcates from the disease-free periodic solution at $\theta \approx 3.866$ through transcritical bifurcation. This positive $T$-periodic solution is stable for $\theta \in(3.866,14.45)$ and unstable for $\theta \in(14.45,+\infty)$. A positive $2 T$-periodic solution bifurcates from the positive $T$-periodic solution at $\theta \approx 14.45$ through flip bifurcation.

The phase space plots for different values of $\theta$ are drawn in Figure 3. Figure 3(a) shows a $T$-periodic solution in system (8) with $\theta=10$. Figure $3(\mathrm{~b})$ shows a $2 T$-periodic solution in system (8) with $\theta=16$. Figure $3(\mathrm{c}$ ) shows a chaotic solution in system (8) with $\theta=54$.

(2) Consider another set of parameters

$$
\begin{aligned}
& A=76.5, \\
& \beta=0.5, \\
& k=0.01, \\
& d=0.5, \\
& T=1, \\
& \gamma=7.3, \\
& \sigma=0.2, \\
& \alpha=30, \\
& \omega=2, \\
& q=0.8 .
\end{aligned}
$$

The bifurcation diagram of system (8) with respect to $\theta$ is presented in Figure 4. System (8) presents complicated dynamics in this case. From Figure 4 , we can see that there exist the chaotic regions and period orbits as the parameter 


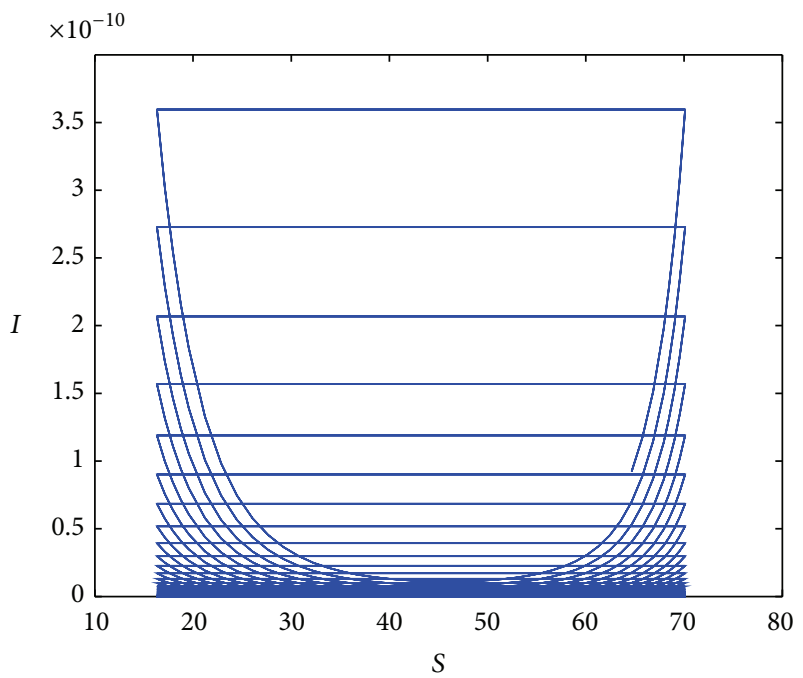

(a) Phase portrait

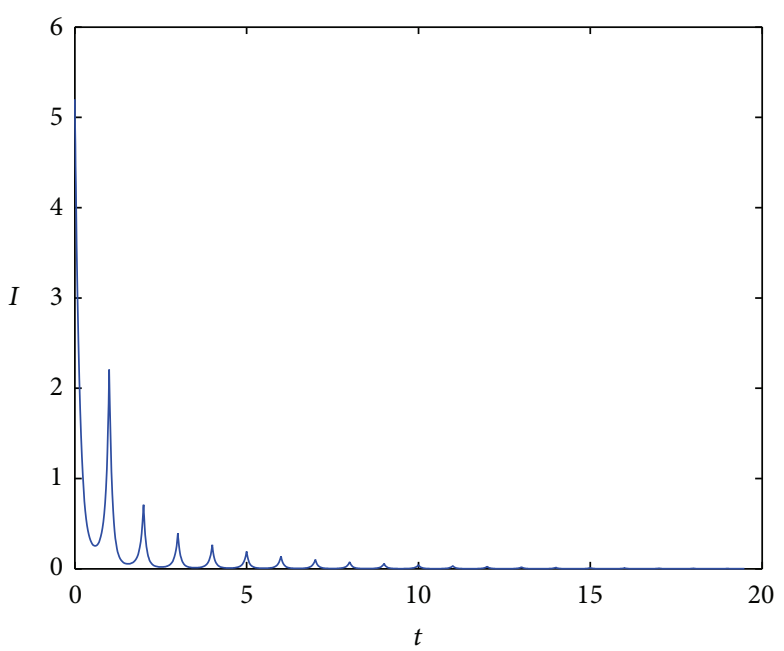

(b) Time series of $I$

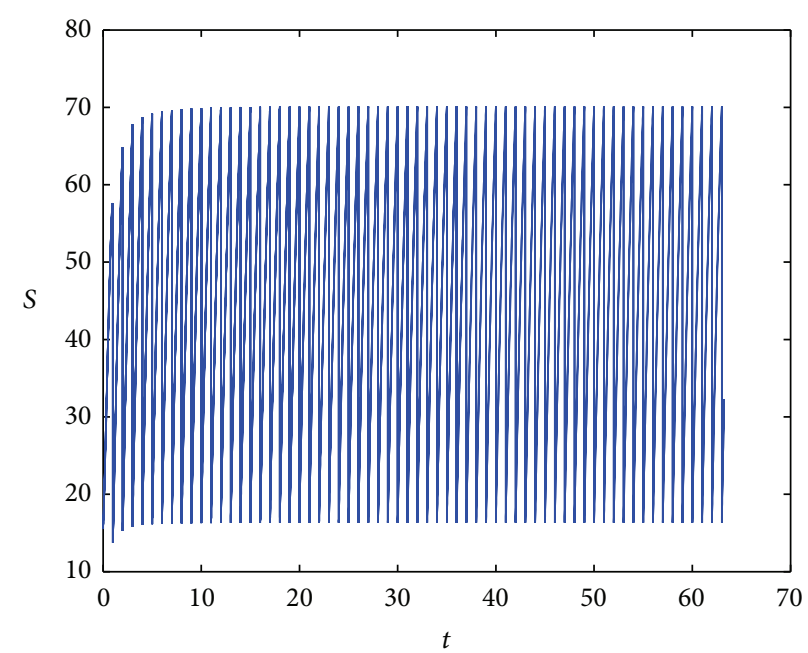

(c) Time series of $S$

FIgURE 1: The stable disease-free periodic solution of system (8) with $\theta=3$.
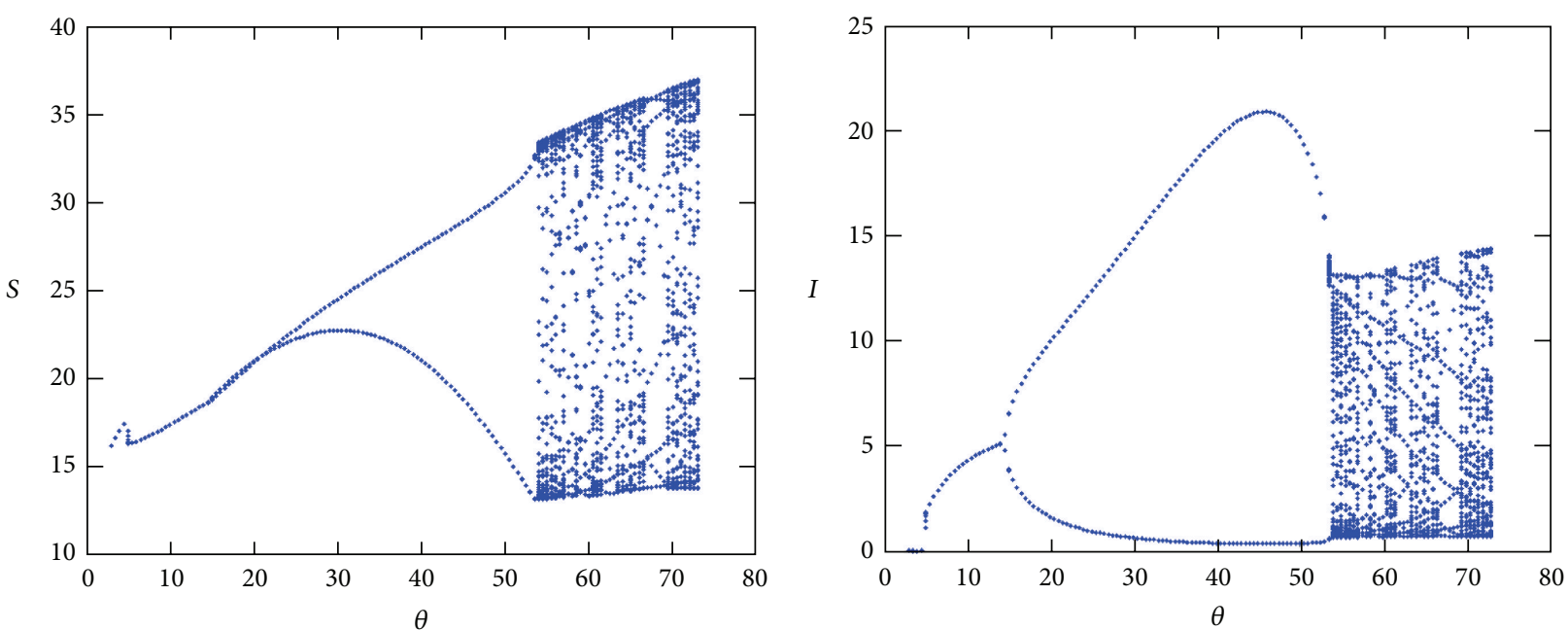

FIGURE 2: Bifurcation diagrams of system (8) with respect to $\theta$ in case (1) of Section 7. 


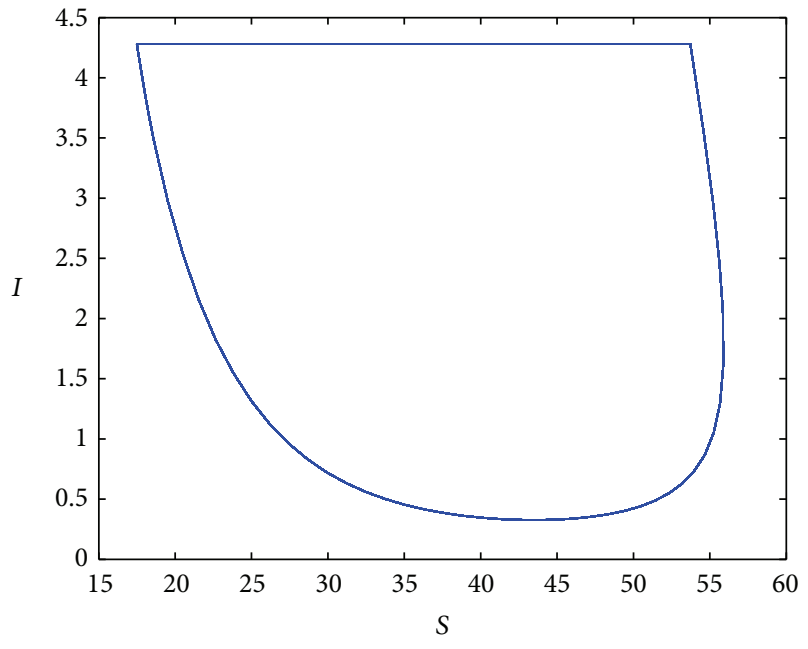

(a) $T$-periodic solution with $\theta=10$

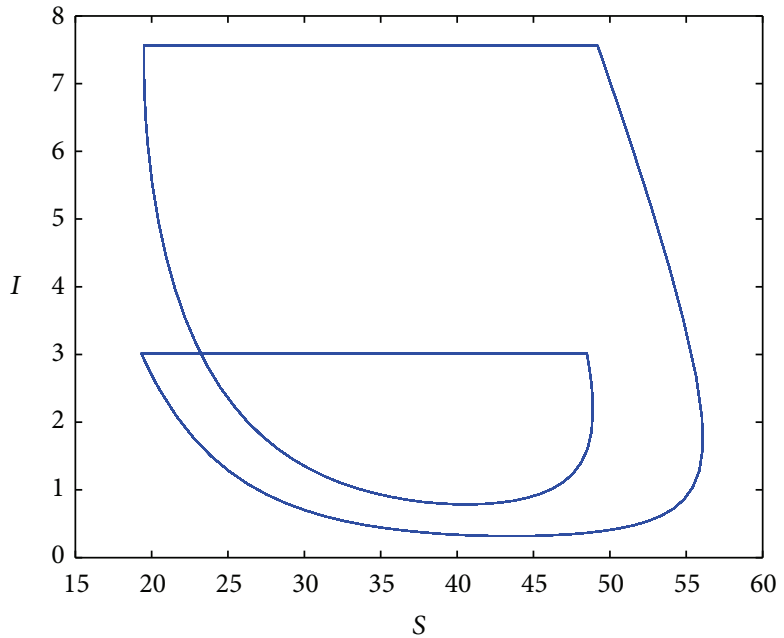

(b) $2 T$-periodic solution with $\theta=16$

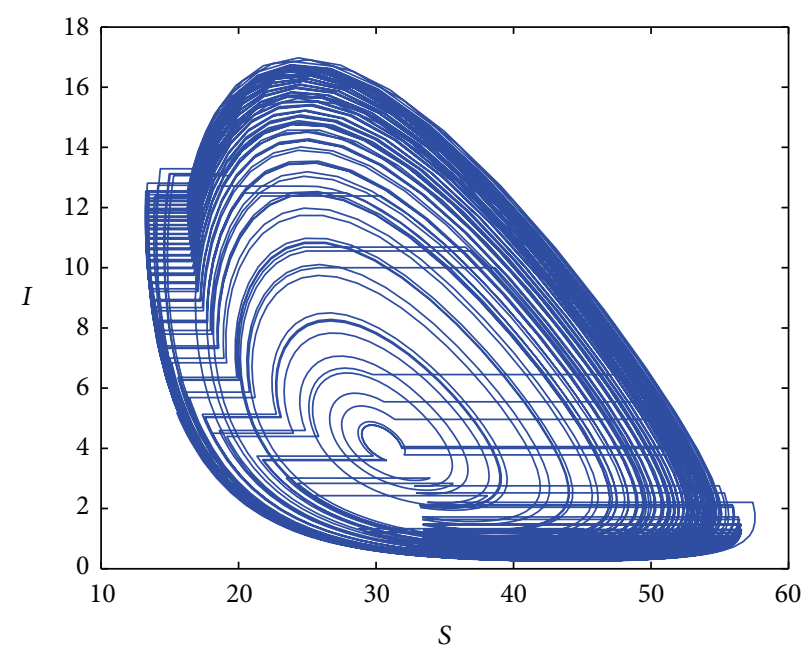

(c) Chaotic solution with $\theta=54$

FIGURE 3: Phase portraits of system (8).

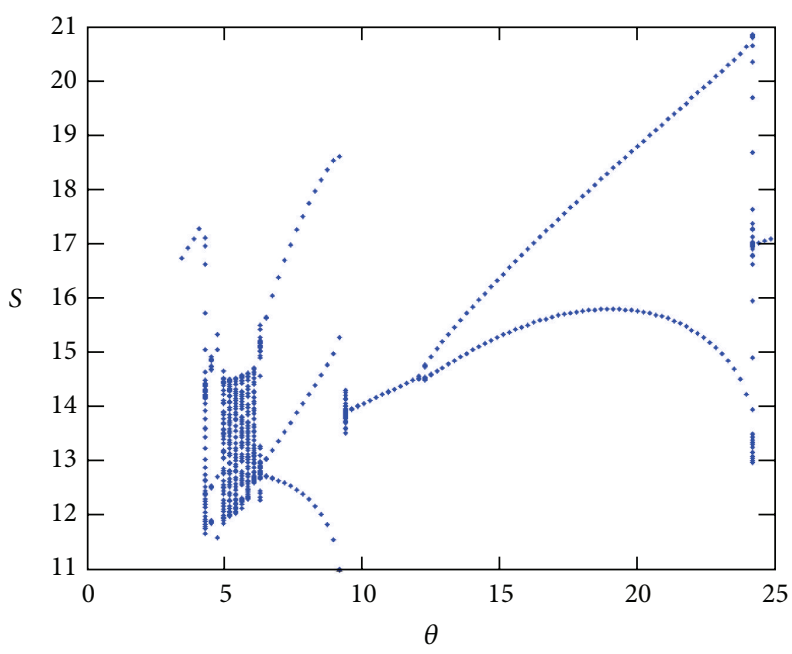

(a)

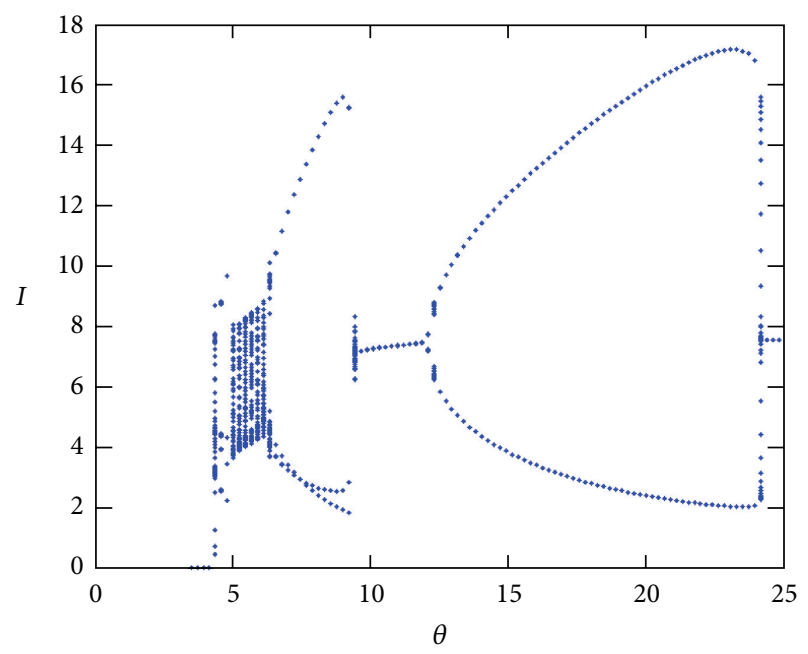

(b)

FIGURE 4: Bifurcation diagrams of system (8) with respect to $\theta$ in case (2) of Section 7. 


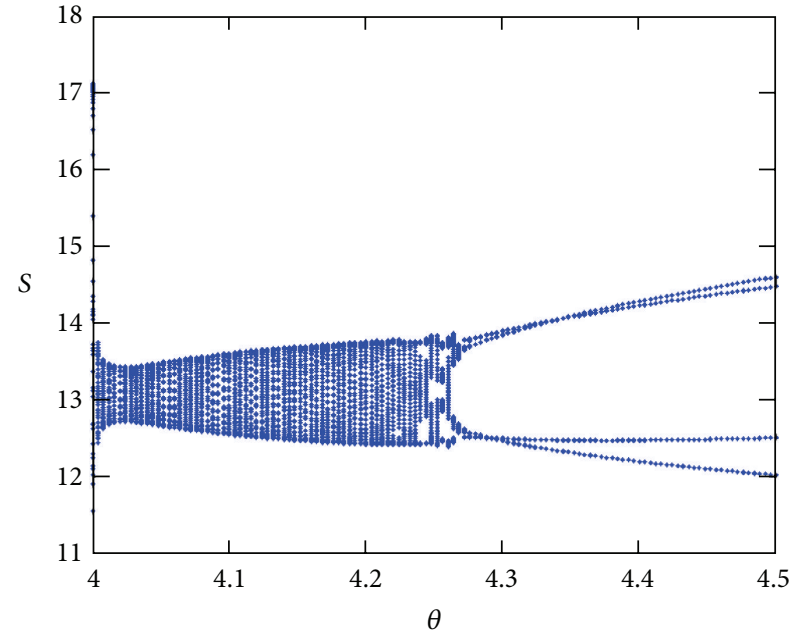

(a) Local amplification of Figure 4 (a) for $\theta \in(4,4.5)$

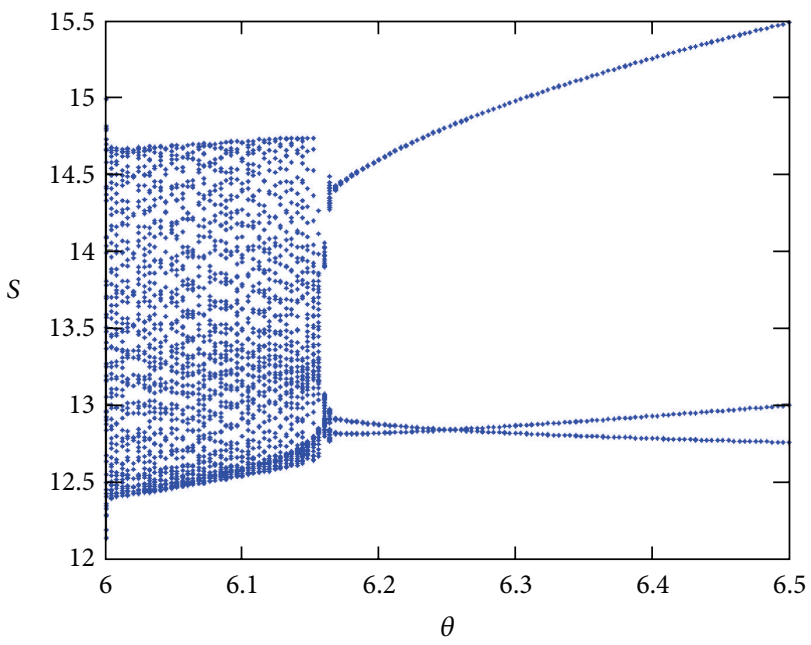

(c) Local amplification of Figure 4(a) for $\theta \in(6,6.5)$

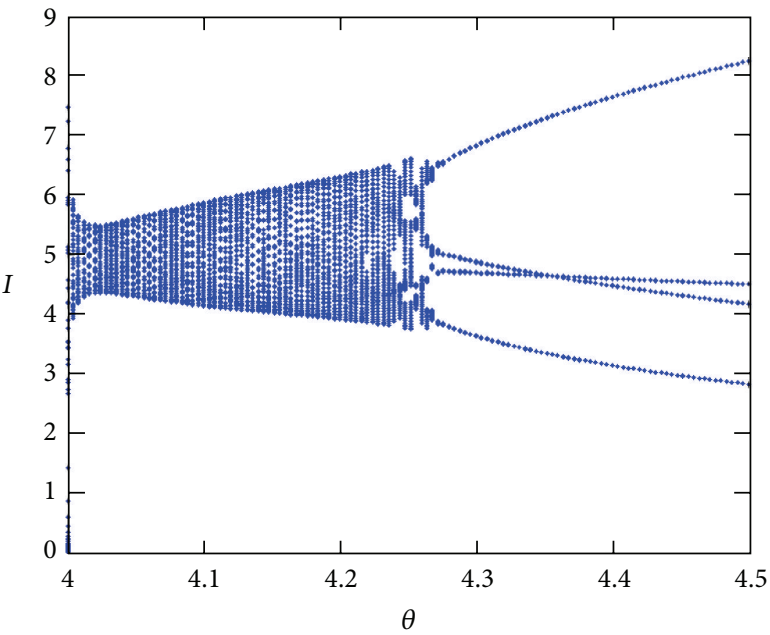

(b) Local amplification of Figure 4(b) for $\theta \in(4,4.5)$

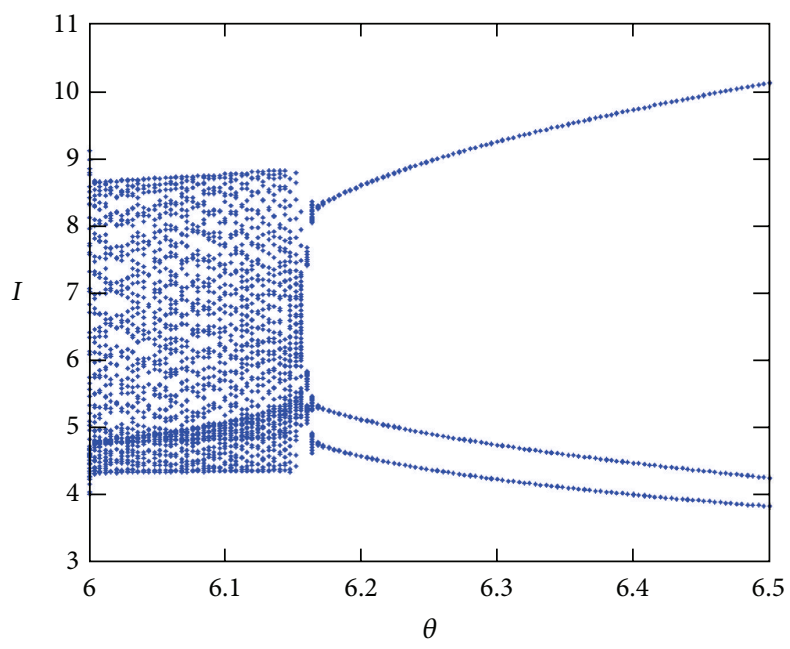

(d) Local amplification of Figure 4(b) for $\theta \in(6,6.5)$

Figure 5: Bifurcation diagrams of system (8) with respect to $\theta$ in case (2) of Section 7.

$\theta$ varies. Figure 4 depicts that there are $T, 2 T, 3 T, 4 T$ periodic windows. From Figure 5, we show the following behaviors: the period-windows within the chaotic regions, the inverse period-doubling bifurcation from $4 T$-periodic orbits to chaos, and the inverse bifurcation from the $3 T$ periodic orbits to chaos.

If we consider $k$ as a parameter, then the bifurcation diagram of system (8) with $\theta=6$ is presented in Figure 6. It is seen from the bifurcation diagram that there is a route from chaos to stable periodic solutions via a cascade of reverse period-doubling bifurcation.

\section{Discussion}

It is well known that one important strategy to control epidemic disease is vaccination. In this paper, an SIR epidemic model with saturated treatment function and nonlinear pulse vaccination is studied. By Remark 4, we may see that the basic reproduction number $R_{0}^{*}$ of system (8) without the pulse vaccination in [16] is greater than the corresponding basic reproduction number $R_{0}$ of system (8). Hence, pulse vaccination may reduce the basic reproduction number. To control the disease, a strategy should reduce the basic reproduction number to below unity. Thus, the introduction of pulse vaccination is helpful in controlling the epidemic diseases. For the control of the epidemic diseases, chaos may cause the diseases to run a higher risk of outbreak due to the unpredictability. Thus, it is necessary to delay or eliminate chaos. It is well known that the flip bifurcations may lead to chaos. So the flip bifurcations should be controlled. We enrich the medical resources (i.e., decrease $\theta$ ) to prevent the flip bifurcations. According to Theorem 11, we may reduce $\theta$ below $\theta_{1}$. Then, the flip bifurcations can be eliminated. In addition, the flip bifurcation will not occur if the stability of the epidemic periodic solutions is not changed. Thus, we may also eliminate the flip bifurcation using Theorem 9. This prevents disease outbreaks. In the following, we eradicate the disease by means of preventing the transcritical bifurcations. 

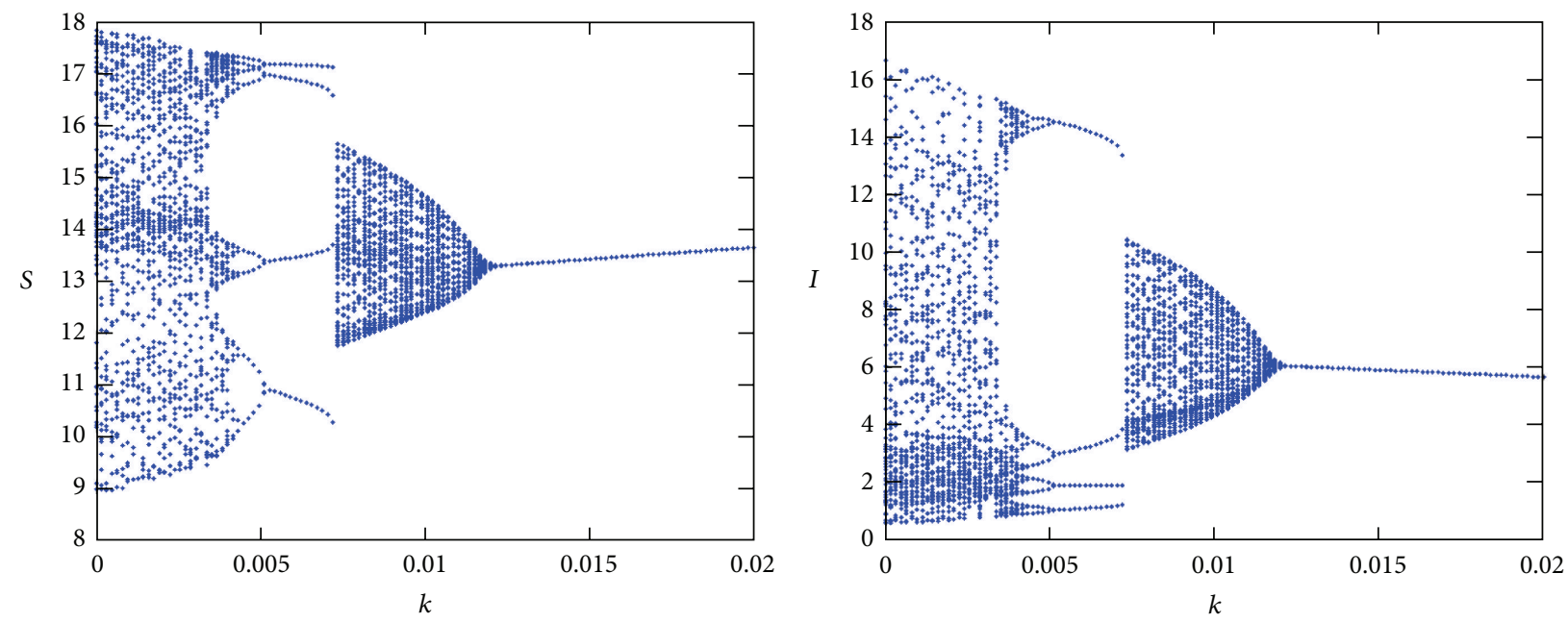

FIGURE 6: Bifurcation diagrams of system (8) with respect to $k$ in case (2) of Section 7.

By Theorem 8, we should consider two cases. For case (1) of Theorem 8 , we may reduce $\theta$ below $\theta_{0}$ (i.e., $R_{0}<1$ ). Then disease eradication may be obtained. However, this might not be sufficient to eliminate the disease for case (2) of Theorem 8 by reducing $\theta$ below $\theta_{0}$ (i.e., $R_{0}<1$ ) since a backward bifurcation occurs. Therefore, we have to identify the critical contact value $\theta_{*}<\theta_{0}$ such that there do not exist the epidemic periodic solutions for $\theta<\theta_{*}$. We may also reduce $R_{1}$ below 1 to eliminate the disease using Theorem 5 .

In addition, by Figure 6 , we find that the parameter $k$ has an impact on dynamical behaviours of system (8). For $k>$ 0.0132 , chaos will not occur from Figure 6 . Hence, we may conclude whether the chaos occurs or not by the value of $k$. Then we may apply the corresponding control strategies.

\section{Competing Interests}

The authors declare that there is no conflict of interests regarding the publication of this paper.

\section{Acknowledgments}

This work is supported by the National Natural Foundation of China (11271371, 51479215).

\section{References}

[1] X. Zhang and X. Liu, "Backward bifurcation of an epidemic model with saturated treatment function," Journal of Mathematical Analysis and Applications, vol. 348, no. 1, pp. 433-443, 2008.

[2] W. Wang and S. Ruan, "Bifurcations in an epidemic model with constant removal rate of the infectives," Journal of Mathematical Analysis and Applications, vol. 291, no. 2, pp. 775-793, 2004.

[3] Z. Zhang and Y. Suo, "Qualitative analysis of a SIR epidemic model with saturated treatment rate," Journal of Applied Mathematics and Computing, vol. 34, no. 1, pp. 177-194, 2010.

[4] H. Khan, R. N. Mohapatra, K. Vajravelu, and S. J. Liao, "The explicit series solution of SIR and SIS epidemic models," Applied
Mathematics and Computation, vol. 215, no. 2, pp. 653-669, 2009.

[5] Z. Hu, W. Ma, and S. Ruan, "Analysis of SIR epidemic models with nonlinear incidence rate and treatment," Mathematical Biosciences, vol. 238, no. 1, pp. 12-20, 2012.

[6] V. Lakshmikantham, D. D. Bainov, and P. S. Simeonov, Theory of Impulsive Differential Equations, vol. 6 of Series in Modern Applied Mathematics, World Scientific, Singapore, 1989.

[7] D. D. Bainov and P. S. Simeonov, Impulsive Differential Equations: Periodic Solutions and Applications, Longman Scientific \& Technical, New York, NY, USA, 1993.

[8] S. Liu, Y. Pei, C. Li, and L. Chen, "Three kinds of TVS in a SIR epidemic model with saturated infectious force and vertical transmission," Applied Mathematical Modelling, vol. 33, no. 4, pp. 1923-1932, 2009.

[9] X.-B. Zhang, H.-F. Huo, H. Xiang, and X.-Y. Meng, "An SIRS epidemic model with pulse vaccination and non-monotonic incidence rate," Nonlinear Analysis: Hybrid Systems, vol. 8, pp. 13-21, 2013.

[10] G. Jiang and Q. Yang, "Bifurcation analysis in an SIR epidemic model with birth pulse and pulse vaccination," Applied Mathematics and Computation, vol. 215, no. 3, pp. 1035-1046, 2009.

[11] S. Gakkhar and K. Negi, "Pulse vaccination in SIRS epidemic model with non-monotonic incidence rate," Chaos, Solitons \& Fractals, vol. 35, no. 3, pp. 626-638, 2008.

[12] L. Hao, G. Jiang, S. Liu, and L. Ling, "Global dynamics of an SIRS epidemic model with saturation incidence," BioSystems, vol. 114, no. 1, pp. 56-63, 2013.

[13] G. Pang and L. Chen, "A delayed SIRS epidemic model with pulse vaccination," Chaos, Solitons \& Fractals, vol. 34, no. 5, pp. 1629-1635, 2007.

[14] W. Wang and S. Ruan, "Bifurcation in an epidemic model with constant removal rate of the infectives," Journal of Mathematical Analysis and Applications, vol. 291, no. 2, pp. 775-793, 2004.

[15] W. Wang, "Backward bifurcation of an epidemic model with treatment," Mathematical Biosciences, vol. 201, no. 1-2, pp. 5871, 2006.

[16] L. Zhou and M. Fan, "Dynamics of an SIR epidemic model with limited medical resources revisited," Nonlinear Analysis: Real World Applications, vol. 13, no. 1, pp. 312-324, 2012. 
[17] V. Capasso and G. Serio, "A generalization of the KermackMcKendrick deterministic epidemic model," Mathematical Biosciences, vol. 42, no. 1-2, pp. 43-61, 1978.

[18] W. Zhao, J. Li, and X. Meng, "Dynamical analysis of SIR epidemic model with nonlinear pulse vaccination and lifelong immunity," Discrete Dynamics in Nature and Society, vol. 2015, Article ID 848623, 10 pages, 2015.

[19] J. Guckenheimer and P. Holmes, Nonlinear Oscillations, Dynamical Systems, and Bifurcations of Vector Fields, vol. 42 of Applied Mathematical Sciences, Springer, New York, NY, USA, 1983. 


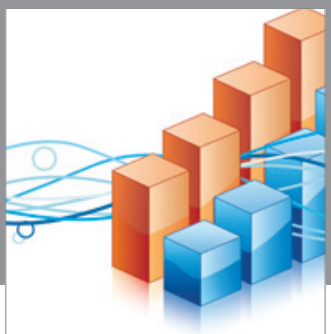

Advances in

Operations Research

vatem alat4

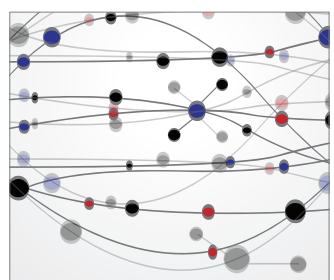

\section{The Scientific} World Journal
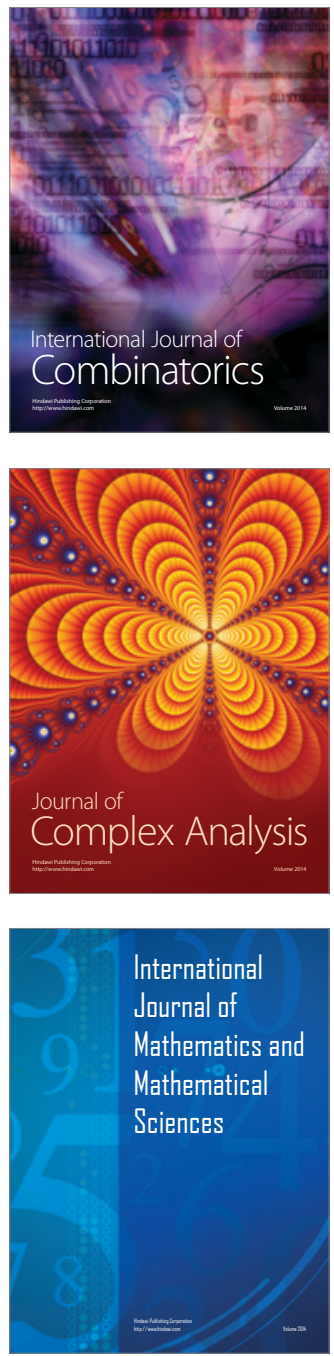
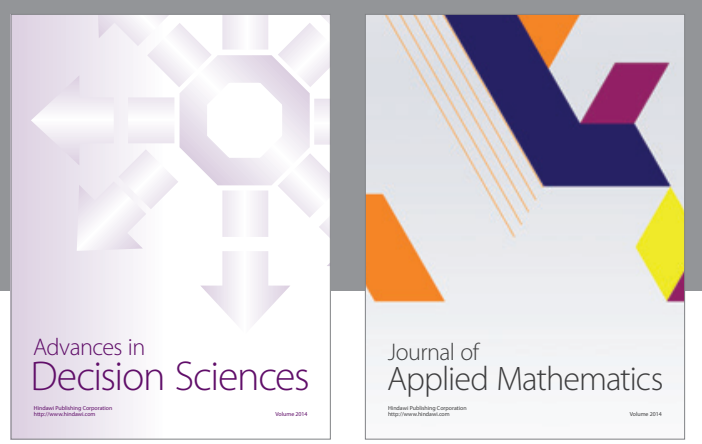

Algebra

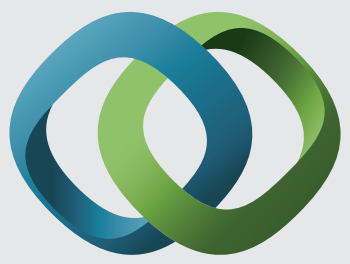

\section{Hindawi}

Submit your manuscripts at

http://www.hindawi.com
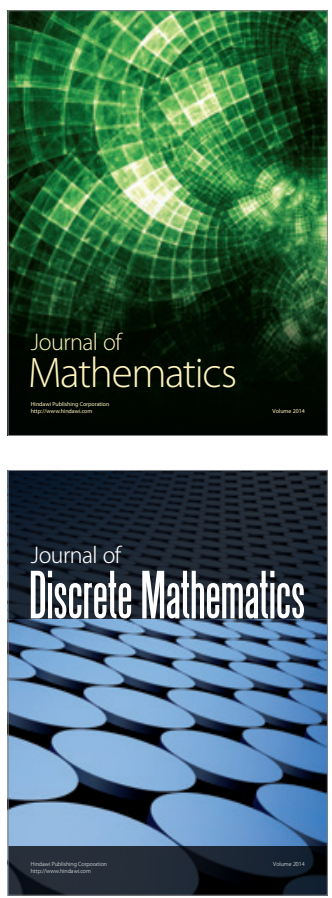

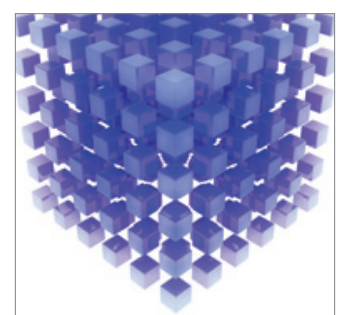

Mathematical Problems in Engineering
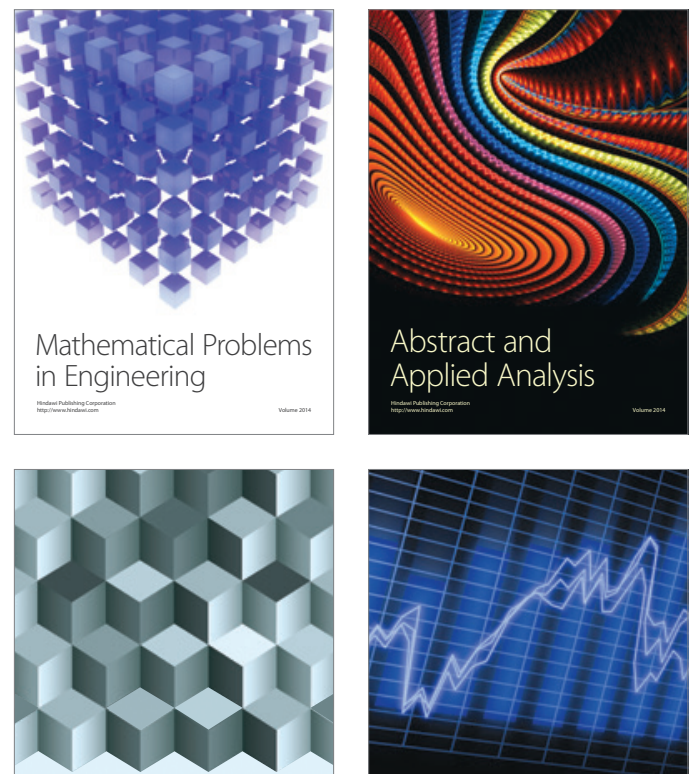

Journal of

Function Spaces

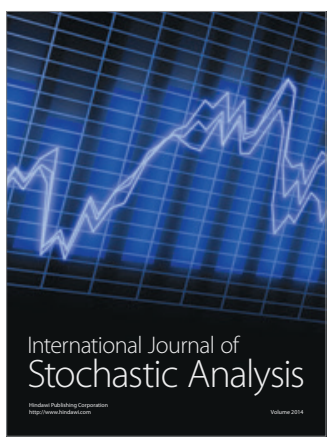

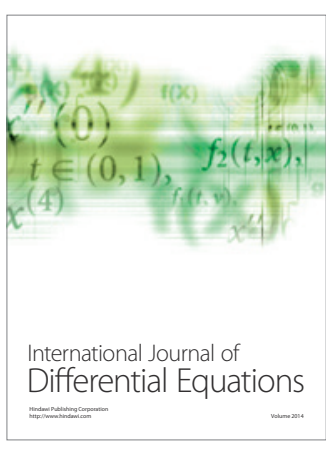
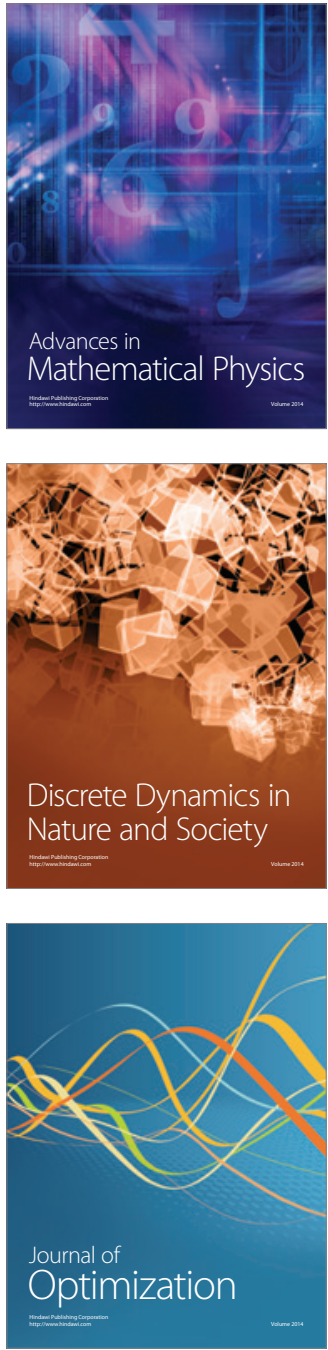